\title{
Birgit Mahnkopf
}

\section{Formel 1 der neuen Sozialdemokratie: Gerechtigkeit durch Ungleichheit. Zur Neuinterpretation der sozialen Frage im globalen Kapitalismus}

Mehr als ein Jahrhundert lang bestand die „Alchimistenfunktion der Sozialdemokratie“ (Jean Ziegler) darin, Angst vor dem Kommunismus in sozialen Fortschritt zu verwandeln. Spätestens seit 1989 schreckt jedoch kein „Gespenst des Kommunismus" mehr und daher scheint die historische Funktion der Sozialdemokratie entbehrlich geworden. Seither wird die Idee eines sozialen Fortschritts, welcher sich an der Verminderung sozialer Ungleichheit erweisen soll, als zutiefst unmodern verworfen - und dies nicht allein durch den siegreichen Liberalismus, der sich heute als eine „realisierte Utopie“ (Karl Polanyi) ohne Alternative versteht. Diese Sichtweise haben sich auch „moderne Sozialdemokraten“ zu eigen gemacht - unter dem Banner eines „dritten Weges“, der auch als „modernes Regieren im 21. Jahrhundert“ propagiert wird.

\section{Eine „mentale Kolonialisierung” der Sozialdemokratie?}

Moderne Sozialdemokraten, welche für die „Sozialarbeiterfraktion in Partei und Gewerkschaften“ (Streeck 2000) nur Hohn und Spott übrig haben, verstehen ihre programmatische Erneuerung als zukunftsweisenden Pfad zwischen dem Modernisierungskurs des Neoliberalismus, der alles auf die Karte ungezügelter Märkte setzt, und einer „bürokratisch denkenden Staatsfixiertheit" (Gerhard Schröder). Gemeint ist mit dem zweiten „Irrweg“ der Sozialstaat der 70er Jahre, den es so wie im "goldenen Zeitalter des Kapitalismus“ ja schon seit den 80er Jahren nicht mehr gibt.

Faktisch aber zielt die Programmatik des „Dritten Weges“ erstens auf eine Apologie der ökonomischen Globalisierung. Diese gilt als eine positive und durch politische Entscheidungen nicht beeinflußbare Entwicklung und verlangt nach einer Neubewertung der Rolle des Staates. Weder der Schutz der Bürger vor den Kräften des Marktes noch die Einhegung der Globalisie- 
rungsprozesse durch Rahmen- und Regelsetzung sollen fortan zentrale Aufgabe von Staat und Politik sein. Die Politik muß sich, so Anthony Giddens, der intellektuelle Stichwortgeber der Debatte, pragmatisch auf die Welt des Kapitalismus einlassen, denn „der Kapitalismus ist das einzige was wir haben“ (Giddens1999a). Daraus folgt, daß die Politik keiner eigenständigen Logik folgen kann, sondern in erster Linie die Steuerungsfähigkeit von Märkten ergänzen und verbessern muß, diese aber keineswegs behindern darf (Giddens 1999b, 2000 sowie zur Kritik u. a. Mahnkopf 2000, Wiethold 2000). Aufgabe des Staates ist es demnach, die Bürger zur Selbsthilfe zu „aktivieren“, sie „marktfähig“ zu machen und all diejenigen Interessen zu „moderieren“, die sich (mit starker Stimme) zu artikulieren wissen und dabei für globalisierungstaugliche Ziele eintreten.

Mit der adaptiven Haltung, die die Modernisten des „Dritten Weges“ gegenüber der Globalisierung einnehmen, verbindet sich zweitens eine Art freiwilliger „Washington Consensus“, d. h. eine Fixierung auf solche politische Maßnahmen, die mit finanzieller Stabilität und sinkenden Steuerlasten vereinbar sind. Dies impliziert den Vorrang von Inflationsvermeidung vor Beschäftigungsausweitung, den Abbau staatlicher Verschuldung vor dem Ausbau- oder auch nur dem Erhalt von sozialen Sicherungssystemen. Im Kern geht es darum, den Währungs- und Steuerraum für mobile Produktionsfaktoren - die Besitzer von Sach- und Geldkapital und die Besitzer von begehrten „Humanressourcen“ - dadurch attraktiv zu machen und zu halten, daß der Vermögenssicherung oberste Priorität eingeräumt wird. Billigend in Kauf genommen wird dabei, daß dies auf Kosten der Möglichkeiten von „immobilen Produktionsfaktoren" geht, ihre Einkommen zu steigern oder auch nur zu sichern. Diese Prioritätensetzung erklärt im übrigen auch die unverhohlene Sympathie „moderner Sozialdemokraten“ für (innovative) Unternehmen, Finanzdienstleister und "Symbolanalytiker" (R. Reich) der unterschiedlichen Couleur. Politisch äußert sich dies in dem Vorrang, der einer Beschleunigung des Innovationstempos in „high tech“-Sektoren der Wirtschaft - gegenüber einer Förderung von (kleinen und mittleren) Unternehmen der „old economy" - eingeräumt wird; in der ideologischen Verklärung von Flexibilisierung und Mobilität zu neuen Sekundärtugenden, die mit der zwieschlächtigen Gestalt des „neuen Selbständigen“ zum gesellschaftlichen Leitbild avancieren; in sozialpolitischen Reformen, die Tendenzen der Individualisierung verstärken - insbesondere durch die Neuausrichtung der Altersversorgung auf individuelle Kapitalbildung (vgl. dazu Mahnkopf 2000).

Drittens markiert die Programmatik des „Dritten Weges“ nicht nur Absetzbewegungen vom Modell des „rheinischen Kapitalismus“ und vom keynesianischen Wohlfahrtsstaat der Nachkriegsära sondern zugleich auch den Abschied der Sozialdemokratie von der Arbeiterbewegung: Um eine sozialstrukturell gesehen eher kleine „neue Mitte“ von Symbolanalytikern zu gewinnen, 
muß die Sozialdemokratie - so noch einmal Anthony Giddens - „von ihrer Fixierung auf die sozial Schwächeren“ lassen. Daher ist es nur folgerichtig, wenn weder der deutsche Bundeskanzler noch der britische Premierminister einen Hehl daraus machen, daß die Gewerkschaften für sie eine Lobby wie jede andere sind. Im übrigen versuchen sie, den historischen Bruch mit den Gewerkschaften durch einen angebotsorientierten Neokorporatismus zu kaschieren - dessen deutsche Variante heißt „Bündnis für Arbeit, Ausbildung und Wettbewerbsfähigkeit“. Mit wettbewerbspolitischen „Sozialpakten“, wie sie derzeit überall in Europa geschmiedet werden, läßt sich eine direkte Konfrontation von gewerkschaftlichen Forderungen und Regierungspolitik vermeiden. Ganz nebenbei werden die Gewerkschaften dabei außer Gefecht gesetzt - weil sie ihre ohnehin stark angegriffene Konfliktfähigkeit nicht mehr zur Geltung bringen können. Jedenfalls müssen sich in Zukunft die deutschen (wie zuvor die britischen) Gewerkschaften daran gewöhnen, ohne jeden „politischen Arm“ auszukommen, scheinen doch Bündnisgrüne und PDS weder willens noch fähig, die Nachfolge der SPD anzutreten (vgl. Urban 2000: 31). Für die Steigerung der internationalen Wettbewerbsfähigkeit von Unternehmen, die im hilflosen Schulterschluß mit der regierenden Sozialdemokratie angestrebt wird, mag dies vorteilhaft sein. Zwar streben die Propagandisten des „Dritten Weges“ eine Steigerung der Wettbewerbsfähigkeit vornehmlich über eine Senkung der Lohnstückkosten an, die durch eine Erhöhung der Arbeitsproduktivität erreicht wird; opportun sind inzwischen jedoch auch Lohnsenkungen, die über eine Reduzierung der Lohnnebenkosten, also der sozialen Lohnbestandteile realisiert werden. Nur die „Modernisierer" in den gewerkschaftlichen Reihen wollen dies noch immer nicht wahrhaben (vgl. dazu Lang 2000).

Allen beschönigenden und aus gutem Grunde zweideutigen Floskeln eines „Dritten Weges“ zum Trotz erweist sich der Modernisierungskurs der Sozialdemokratie als Einbürgerung neoliberalen Gedankenguts. Exemplarisch läßt sich dies an dem vielstimmigen Plädoyer für einen „zeitgemäßen Gerechtigkeitsbegriff“ zeigen, mit dem im Frühjahr 2000 die öffentliche Debatte über ein neues Grundsatzprogramm der SPD eröffnet wurde (vgl. dazu: http://www.spd.de/events/grundwerte/html. Ganz offensichtlich findet hier ein Prozeß „mentaler Kolonialisierung“ (Bourdieu/Wacquant 2000) statt; Michael Ehrke von der Friedrich-Ebert-Stiftung schreibt schnörkelloser von einer „Amerikanisierung der Sozialdemokratie“ (Ehrke 1999). Wie das Plädoyer für den Rückzug des Staates aus der Wirtschaft, das Hohelied auf die befreienden Wirkungen der Deregulierung von Märkten, die Beschwörung von „Flexibilität“ und „employability“, das moralisierende Lob der „individuellen Verantwortung“ oder die postmodernen Diskurse über „kulturelle Anerkennung“, „Multikulturalismus“ und „Identitätspolitik“ gehört auch die neoliberale Fassung der Gerechtigkeitsidee zu einem „neuen globalen Begriffska- 
non", dessen Herkunft Pierre Bourdieu und Loic Wacquant nicht ganz zu unrecht in den Besonderheiten der US-amerikanischen Gesellschaft und ihrer Universitäten vermuten: Wahrnehmungskategorien, die den Sozialstrukturen der USA entsprechen, werden „von vorgeblich neutralen Instanzen übernommen, angefangen von den großen internationalen Organisationen wie der Weltbank oder der OECD, über die konservativen ,think tanks' bis hin zu den großen Medien, die diese Lingua franca rastlos weiterverbreiten, deren Phrasen den eiligen Leittartiklern und geschäftigen Spezialisten kulturellen Import-Exports die Illusion vermitteln, an der Spitze der Modernisierung zu marschieren“ (Bourdieu/ Wacquant 2000; vgl. zur „mentalen Kolonialisierung" der Sozialdemokratie auch Zeuner 2000).

Die Eigentümlichkeit des Neoliberalismus besteht darin, daß es sich dabei um politische Ideen handelt, welche die Realitäten, die sie zu beschreiben beanspruchen, im Sinne einer sich selbst erfüllenden Prophezeiung erst hervorbringen: Zunächst identifizieren Politiker, Publizisten und medienpräsente Intellektuelle - unter Verweis auf Projektionen, die Entwicklungsannahmen quantifizieren und/oder gegebene Daten hochrechnen - gesellschaftliche Entwicklungen als interessenfreie, quasi-natürliche Phänomene. Danach wird den zuvor identifizierten „Trends“, die genaugenommen eher Annahmen über eine zukünftige Entwicklung sind, durch politische Entscheidungen zum Durchbruch verholfen. So gesehen geht es bei der aktuellen Debatte über die sozialdemokratischen "Grundwerte: Gerechtigkeit, Freiheit und Solidarität“ darum, ob der Neoliberalismus in Europa die ideologische Hegemonie behaupten kann - über die Filter- und Pufferwirkung eines „zweiten Revisionismus der Sozialdemokratie“ (vgl. Ehrke 1999). Denn es macht einen Unterschied, ob die größer gewordenen sozialen Ungleichheiten von jener Partei gerechtfertigt werden, die einmal die der „kleinen Leute“ war oder von Vertretern konservativer und liberaler Parteien - die deutschen Bündnisgrünen dürfen inzwischen ohne Vorbehalte zum letzteren Typus gerechnet werden: Wenn schließlich auch die SPD auf die tiefer werdende soziale Spaltung der Gesellschaft mit einer Programmatik der radikalisierten Flexibilisierung und Individualisierung von Erwerbsarbeit reagiert und damit marktkonformes Denken in tendenziell allen Lebensbereichen befördern will, so bedeutet dies, daß die „Modernisierungsverlierer" ohne jede politische Stimme bleiben - so lange, bis der Rechtspopulismus ihr verletztes Gerechtigkeitsempfinden für seine Zwecke zu instrumentalisieren weiß.

Vor einiger Zeit hat die Unternehmensberatungsfirma Anderson Consulting ein „best“ und ein „worst case“-Szenario zur Zukunft Europas im Jahre 2008 entworfen (vgl. Blomert 1999): In der wünschenswerten Variante avancieren Individualismus und Unternehmergeist zu den treibenden kulturellen Kräfte. Weil die Unternehmen jedoch immer weniger Steuern zahlen, die Arbeitnehmer verarmt sein werden und die privatisierten Versorgungsbetriebe kein 
Geld mehr in die öffentlichen Kassen spülen, kann der Staat nur noch mit geringen Steuereinnahmen rechnen. Das zwingt die Unternehmen, die defizitäre Bildung in eigener Regie zu betreiben. Allerdings werden unter den Bedingungen eines zum Nachtwächterstaat degradierten Wohlfahrtsstaates die Kosten für die Überwachung von Betrieben, sozial klar segmentierten Einkaufszonen und Siedlungen der Oberschicht kräftig ansteigen. Aus der Perspektive von Anderson Consulting handelt es sich dabei allerdings um hinnehmbare Nachteile, die mit der Durchsetzung der US-amerikanischen Kapitalismusvariante verbunden wären. Denn die Alternative verheißt noch Schlimmeres: Im „worst case“-Szenario einer demokratisch regulierten „Festung Europa" beschränken die politischen Institutionen Europas die ökonomischen Kräfte. Fusionen und Übernahmen unterliegen einer strengen Kontrolle und werden sogar im Hinblick auf ihre Beschäftigungsfolgen überprüft. Es würde Löhne oberhalb der Inflationsrate geben, hohe Steuern, hohe Sozialabgaben und strenge Auflagen für die Betriebe, so daß diese gezwungen sind, innerhalb eines stark kontrollierten Umfeldes zu agieren. Hohe öffentliche Investitionen, langfristige Planungshorizonte für die Unternehmen, (nur) langfristige Renditen und einiges mehr bilden aus Sicht der weltweit agierenden Unternehmensberatung die Facetten der unerfreulichsten, aber eher unwahrscheinlichen Zukunft Europas. Die Entscheidung über diese beiden Varianten wird derzeit in Deutschland ausgefochten - in und durch die deutsche Sozialdemokratie.

\section{Die Neuinterpretation der sozialen Frage}

Mitglieder und Wähler sind verwirrt, die Presse zeigt sich angenehm überrascht und die Industrie frohlockt: Im April 2000 eröffnet die SPD eine Debatte über ihr neues Grundsatzprogramm mit einem Forum zum „Grundwert Gerechtigkeit“. Dabei rückt sie die Frage ins Zentrum, wieviel Gleichheit die Gerechtigkeit erfordere und wieviel Freiheit für ökonomische Dynamik unabdingbar sei. Bei dieser Frage handelt es sich um eine inzwischen leicht angestaubte Denkfigur aus der „Waffenkammer“ des Liberalismus, der von Seiten der politischen Linken in der Vergangenheit entgegengehalten wurde, daß Freiheit und Gleichheit nicht als ein Gegensatz gedacht werden können. Damit verband sich zugleich eine Kritik an dem Gesellschaftsverständnis, das einer solchen Entgegensetzung zugrunde liegt - der Vorstellung einer „Gesellschaft von Individuen“. Zwar handelt es sich dabei um einen Mythos, doch stellt dieser „partikulare, durch den Markt geförderte Interessen zufrieden“ (Lefort 2000). Die Legitimation von partikularen Interessen war bislang die Sache europäischer Sozialdemokraten nicht. Ihr reformistisches Projekt zielte vielmehr darauf $a b$, durch eine Politisierung der sekundären Verteilung des erwirtschafteten Reichtums eine Korrektur der primären Verteilung durch den 
Markt und eine Neutralisierung des gesellschaftlichen Konflikts zwischen Kapital und Arbeit herbeizuführen.

So liegt es keineswegs auf der Hand, weshalb ein neues Parteiprogramm für das 21. Jahrhundert darauf abzielen müßte, den Entfaltungsspielraum individueller Freiheitsrechte in der Sphäre der Ökonomie (und daher des privaten Unternehmertums) zu vergrößern. Zumal deswegen nicht, weil neue Managementkonzepte der „operativen Dezentralisierung“ (outsourcing, Profit-CenterOrganisastion, Gruppenarbeit) die mit der Kritik an hierarchischen und bürokratischen Organisationsformen verbundene „Befreiungs-Forderung“ nach „Autonomie“ längst in einem $Z$ wang zu individueller Verantwortung und Selbstmanagement verkehrt haben - um den Preis einer Verminderung von Schutzrechten und der Sicherheit von Arbeitsplätzen. Unter den Bedingungen globalen Wettbewerbs erhält das liberale Plädoyer für die Wahrnehmung und Ausdehnung individueller Freiheitsrechte die Bedeutung, daß alle lokalen Bande und Zugehörigkeiten als Quelle von Erstarrung zu zerreißen seien. Daher wird die Aufforderung zu unbegrenzter und unbestimmter „Autonomie“ von vielen Menschen keineswegs als Aufruf zur „Befreiung“ erlebt sondern als Ankündigung einer „Prekarisierung“ ihrer Erwerbs- und Lebensbedingungen (vgl. Boltanski/Chiapello 1999). Weil jedoch äußerst strittig ist, was heute Kennzeichen einer „Befreiung“ und was Kennzeichen neuer Knechtschaft wäre, verweist die Entgegensetzung von Freiheit und Gleichheit nicht so sehr auf eine problematische Begrenzung individueller Freiheitsrechte in der Sphäre ökonomischer Verhaltensweisen sondern eher darauf, daß das politische Ziel größtmöglicher Gleichheit (nun auch) von Sozialdemokraten in Frage gestellt wird.

\section{Der Verteilungskompromiß der Nachkriegsära}

In seinem Kern bestand das reformistische Projekt der Sozialdemokratie darin, die zentrale Achse des gesellschaftlichen Konflikts um die Macht aus der Sphäre der Produktion in die von Verteilung und Konsum zu verschieben. Auf diese Weise entwickelte sich die Arbeiterbewegung, einst Trägerin eines alternativen Gesellschaftsmodells, zu einem Motor der gesellschaftlichen und wirtschaftlichen Entwicklung im 20. Jahrhundert. Über Reallohnerhöhungen wurden die Beschäftigten an den Produktivitätsfortschritten beteiligt. Dabei erhielt die Umverteilung eines Teils des erwirtschafteten Reichtums zugunsten des Faktors Arbeit die Funktion, die Gesamtnachfrage zu stützen, mithin eine an die Entwicklung der Produktivkräfte angepaßte Ausdehnung des Marktes zu ermöglichen. Das war auch der Grund, weshalb sich im „goldenen Zeitalter" des Fordismus, das zugleich ein "sozialdemokratisches Zeitalter“ (R. Dahrendorf) war, auch Exponenten aus dem liberal-konservativen Lager auf das politische Ziel der Verteilungsgerechtigkeit verständigen konnten. 
Denn so lange der zu verteilende Kuchen beständig größer wurde, erhielten alle - im Vergleich zu früheren, „normalen“ Zeiten des Wachstums - ein sehr viel größeres Stück, ohne daß die Frage der Umverteilung von oben nach unten mit einschneidenden Konsequenzen verbunden gewesen wäre. Eine zumeist bescheidene, von den Gewerkschaften in zum Teil harten Tarifauseinandersetzungen erstrittene „Umverteilungskomponente“ sorgte dafür, daß der Anteil der Lohneinkommen am gesellschaftlichen Gesamteinkommen und die Sozialeinkommen stieg. Dadurch wurde ein „virtuoser Kreislauf von Wachstum und sozialer Gerechtigkeit“ (Revelli 1999: 125ff) in Gang gesetzt: Wegen ihrer positiven Effekte auf die Binnennachfrage wird die Umverteilung $\mathrm{zu}$ einer wichtigen Bedingung für weiteres Wachstum und zugleich kann ein Teil des erwirtschafteten Überschusses in soziale Sicherheit investiert werden. Dabei rückt die Verteilung des Überschusses schrittweise in die Verantwortung des Staates, mit der Folge, daß einerseits dessen Aufgaben und Zuständigkeiten rasch wachsen und zweitens Verteilungsansprüche zunehmend an den Staat gestellt werden, die Frage der Verteilung daher eine starke Politisierung erfährt.

Insbesondere in seiner deutschen Variante basierte das sozialdemokratische Konzept von Verteilungsgerechtigkeit auf einem ökonomisch begründeten „Produktivitätspakt“ zwischen Arbeit und Kapital, der durch staatliche Sozialpolitik flankiert wurde. Das „Modell Deutschland“ wurde zu einem Synonym für die Verbindung von internationaler Wettbewerbsfähigkeit der Wirtschaft mit vergleichsweise geringer inländischer Lohnungleichheit und einem relativ dichten Netz sozialer Sicherung, welches die Menschen, die durch den Einsatz der „Produktivitätspeitsche“ in die Arbeitslosigkeit gedrängt worden waren, vor einem dauerhaften ökonomischen und sozialen Ausschluß bewahrte (vgl. Mahnkopf 1999a). Mit der Verlangsamung der Wachstumssteigerungen seit Mitte der 70er Jahre, die das Ende vom „Traum immerwährender Prosperität“ (B. Lutz) einleitete, gerät das sozialdemokratische Konzept der Verteilungsgerechtigkeit unter Druck. Dies hat zwar nichts damit zu tun, daß seither zu wenig gesellschaftlicher Reichtum produziert worden wäre - 1999 betrug das private Geldvermögen der Deutschen 6749 Milliarden Mark, damit ist es seit 1992 um mehr als die Hälfte gewachsen. Allerdings ist die Produktivität, obwohl ihre Zuwächse in den Industrieländern seit den 60er Jahren sinken, um einiges schneller gestiegen als das industrielle Wachstum (pro Kopf); in Deutschland konnte das BIP des Jahres 1996 mit etwa der Hälfte der Arbeit erzeugt werden, die noch 1970 nötig war. Die Folgen sind bekannt: Es sinkt das Arbeitsvolumen, die Massenarbeitslosigkeit wird zu einem strukturellen Merkmal des sozio-ökonomischen Gefüges.

Darauf reagiert die Sozialdemokratie mit einer „zweiten Revision“ ihrer politischen Identität: Sie verabschiedet sich von ihrem Engagement für die Benachteiligten und von dem Versuch, wirtschaftliche gesellschaftliche Entwick- 
lungsprozesse gemäß rationalen Prinzipien steuern zu wollen (Ehrke 1999). Dabei nimmt sie jedoch in Kauf, daß die ohnehin schon recht morschen sozialen Fundamente des „Hauses der Demokratie“ (R. Dahrendorf) ihre Tragfähigkeit für eine zivile Gesellschaft verlieren. Denn es war die sukzessive Ausweitung von bürgerlichen und politischen Rechten zu sozialen und ,industriellen“ Rechten (Th. H. Marshall), welche die Grundlage für die Zivilisierung und Demokratisierung des post-faschistischen Deutschlands gelegt hatte und der westdeutschen Gesellschaft der Nachkriegszeit eine Distanzierung von deutsch-nationalem Denken ermöglichte (vgl. Seidel 2000). In einem auf sozialen Ausgleich hin konzipierten Sozialstaat konnten die „kleinen Leute“ die Erfahrung existentieller Unsicherheit hinter sich lassen und in den „Genuß“ von Stabilität, Kalkulierbarkeit, Langfristigkeit ihrer Lebensplanung gelangen. Dies waren essentielle Voraussetzungen für die Konsolidierung und Vertiefung der partizipativen Demokratie (vgl. Altvater/Mahnkopf 1999: Kap. 12). Nun aber steht zu befürchten, daß die Politik des „Dritten Weges“, indem sie sich die neoliberale Floskel von den „produktitivätsorientierten Ungleichheiten“" zu eigen macht, dazu beiträgt, die Attraktivität der repräsentativen und partizipativen Demokratie nachhaltig zu schädigen.

\section{Die Entdeckung „produktivitätsorientierter Ungleichheiten”}

Anders als Konservative, Alt-Liberale und Neu-Grüne, für die Gleichheit als regulative Idee von Gesellschaftspolitik nie zum Kernbestand ihrer politischen Identität gehörte, repräsentierten Sozialdemokraten bis vor kurzem noch für breite Gesellschaftsschichten das Ideal, Gerechtigkeit durch eine Verringerung von sozialer Ungleichheit zu erreichen. In der Vergangenheit war das egalitäre Prinzip zumindest insofern konstitutiv für die sozialdemokratische Linke, als diese danach strebte, die „Ungleichheiten etwas gleicher“ werden zu lassen (Noberto Bobbio). Auch wenn heute das Ziel größtmöglicher Gleichheit unter Verdacht gestellt wird, die Eigenheiten der Menschen beseitigen, sie „gleich machen“ zu wollen (vgl. u.a. Dettling 2000), so war doch die einfache Gleichheit, also eine strikte Gleichbehandlung aller Individuen, zu keiner Zeit ein sozialdemokratische Ideal. Gerechtigkeit wurde (nicht nur) in dieser Tradition immer gleichgesetzt mit einer Vergrößerung von Gleichheit. Die Aneignung in der Produktionssphäre ist für Sozialdemokraten schon lange kein Thema mehr und daher wird die „machtbegründete und auf die ultima irratio der Gewalt gestützte Bemächtigung und Instrumentalisierung von Lebensbedingungen und Lebensäußerungen (insbesondere der Arbeit und Arbeitsergebnisse!) Anderer“ (Ritsert 1997: 20) auch nicht mit Fragen der Gerechtigkeit in Zusammenhang gebracht. Längst vergessen ist auch Marxens „Kritik des Gothaer Programm“, wonach der „enge bürgerliche Rechtshorizont“ zugunsten einer Gesellschaftsordnung zu überschreiten 
wäre, die sich das Prinzip „Jeder nach seinen Fähigkeiten, jedem nach seinen Bedürfnissen" auf die Fahnen schreibt (MEW 19: 21).

Die Sozialdemokratie hat sich längst den liberalen Grundsatz des proportionalen Vergeltens zu eigen gemacht. Danach gelten Unterschiede in der Verteilung von Gütern und Ressourcen als legitim, so lange sie in einem spezifischen Bedürfnis, Verdienst oder in Leistungsdifferenzen begründet sind. Gerechtigkeit hat etwas mit dem „rechten“ oder dem „Mittelmaß“ (Aristoteles, Nikomachische Ethik, 5. Buch) zu tun, mit dem Vermeiden eines „Zuviel“ und eines „Zuwenig“, mit dem „Ausgleich“ also. Bei der Verteilung von Lasten und Entschädigungen, Leistungen und Gegenleistungen, Rechten und Pflichten soll ein/e Jede/r bekommen, worauf er oder sie aufgrund gesellschaftlich anerkannter Kriterien einen Anspruch hat (so im Anschluß an Aristoteles auch Hobbes 1959/1939). Es muß daher einen Maßstab geben: für durchschnittliche Leistungen, zumutbare Leistungsanforderungen und für eine Hierarchie von Tätigkeiten, die gesellschaftlich unterschiedlich bewertet werden. Das Prinzip der Leistungsgerechtigkeit verspricht jedem das, was er oder sie „verdient“. Die Funktionsstellen sollen nach ihrer Bedeutung für den gesellschaftlichen Lebenszusammenhang abgestuft und die darauf erbrachten Leistungen entsprechend proportional abgegolten werden.

Während indes der Marktliberalismus gleiche Zugangsbedingungen zu freien Märkten, mithin die Chancengleichheit, immer voraussetzt und daher jede nachträgliche Korrektur von bereits realisierten Ungleichheiten faktisch ausschließt, galten in der sozialdemokratischen Tradition unverschuldete Unterschiede in den Leistungsergebnissen nicht als legitim. Sofern solche Unterschiede der Zufälligkeit der sozialen Herkunft oder der natürlichen Verteilung von Fähigkeiten und Talenten geschuldet waren, sollten Ungleiche eine ungleiche Behandlung erfahren. So gesehen befanden sich Sozialdemokraten stets in Übereinstimmung mit dem Rawlsschen Diktum, wonach die Verteilung von Einkommen, Vermögens, Macht und Verantwortung in der Gesellschaft nicht ausschließlich der „Lotterie der Natur“ (Rawls 1991: 19ff, 81ff) überlassen werden kann. Der Markt ist ungerecht, gerade weil er keine Unterschiede macht: Wer bei der "Lotterie der Natur" oder dank seiner sozialen Herkunft zufällig das „große Los“ gezogen hat, wird vom Markt genauso behandelt, wie diejenigen, die durch ihre natürliche oder soziale Schlechterstellung mit mehr oder weniger schweren „handicaps“ in das marktwirtschaftliche Konkurrenz- und Tauschsystem eintreten. Dadurch werden die bereits realisierten Ungleichheiten reproduziert und verstärkt. Ein gesellschaftliches Verteilungssystem kann sich die Kriterien, nach denen Kooperationsgewinne verteilt werden, aber nicht von einem in verteilungsmoralischer Hinsicht unzurechnungsfähigen Markt vorgeben lassen. Der Markt sorgt noch nicht einmal für eine gerechte Verteilung unter denjenigen Menschen, die ihren Unterhalt selbständig bestreiten können; erst recht kann er nicht für eine ge- 
rechte Versorgung von „nicht-marktfähigen“ Menschen sorgen, also denjenigen Mitgliedern der Gesellschaft, die auf dem Markt nichts anzubieten haben oder aber nur solche Leistungen, die auf dem Markt nicht nachgefragt werden (vgl. Kersting 1998) Die Ungleichbehandlung von Ungleichen ist daher legitim, solange sie moralisch begründet wird, etwa mit der Absicht, Gerechtigkeitsdefizite der Verteilung eines Gutes auszugleichen. Dieses Ziel stand (neben anderen) beispielsweise hinter der sozial-liberalen Bildungsreform der 70er Jahre.

Neu ist an dem Rekurs führender deutscher Sozialdemokraten auf die Gerechtigkeitstheorie des „egalitären Liberalismus“ vor allem die Manier, in der das Rawlssche „Differenzprinzip“ für eine Neuinterpratation der „sozialen Frage“ zum Einsatz kommt. In der Tradition vertragstheoretischen Denkens unterstellt Rawls bekanntermaßen, daß souveräne und vernunftbegabte Individuen in einem hypothetisch angenommen Urzustand - hinter dem „Schleier des Nichtwissens" und damit frei von jeglichen sozialen Bindungen, Interessen und Überzeugungen - nicht nur den Grundsatz gleicher Grundrechte und -pflichten wählen würden, sondern auch einen zweiten Gerechtigkeitsgrundsatz zustimmen würden: Sie würden nichts Ungerechtes an sozialer und wirtschaftlicher Ungleichheit, also an den größeren Vorteilen weniger sehen, wenn sich daraus Vorteile für jeden, insbesondere aber für die schwächsten Mitglieder der Gesellschaft ergeben würden (Rawls 1991, ebenda).

Zwei Einwände gegen diese theoretisch konstituierte Modellsituation sind für eine Kritik des „neuen Gerechtigkeitsdiskurses“, mit dem gegenwärtig ein Allparteienkonsens - unter Ausnahme der PDS - fixiert werden soll, von Bedeutung (vgl. dazu auch Müller-Plantenberg in diesem Heft). Der erste Einwand bezieht sich auf den hypothetischen Urzustand in der Rawlsschen Argumentation, ein zweiter Einwand, auf den erst an späterer Stelle eingegangen wird, auf den (nationalstaatlich) begrenzten Rahmen seiner Gerechtigkeitsgrundsätze: Es ist schwer einzusehen, weshalb Menschen, die sich alle in der gleichen Lage, also einer „fairen Ausgangsposition“ (J. Rawls) befinden, die Ungleichheit wählen sollten und weshalb soziale und ökonomischen Ungleichheiten in einem fiktiven Urzustand irgend jemandem zum Vorteil gereichen sollten. Der Rekurs auf Rawls Differenzprinzip entpuppt sich daher unschwer als ein Versuch, bestehende Ungleichheitsstrukturen außer Streit zu stellen und die Debatte auf eine (gleichmäßige) Verteilung von Vor- und Nachteilen innerhalb derselben zu verlagern.

Im Zentrum der Debatte über den „sozialdemokratische Grundwert Gerechtigkeit“" steht der Verweis auf hypothetische Vorteile Schlechtergestellter, welche aus „begrenzten Ungleichheiten“ resultieren sollen. Dieser Verweis hat die Funktion, ein neoliberales Argument - daß Ungleichheit für wirtschaftliche Effizienz funktional sei - mit einer ganz und gar nicht „modernen“, sondern sehr traditionellen Denkfigur aus dem sozialdemokratischen Instrumen- 
tenkasten zusammen zu spannen - mit dem Postulat, daß Wachstumssteigerung Umverteilung ersetzen kann und soll. Die aus neoliberalen Textbüchern vertraute Formel von der Ungleichheit stiftenden und Ungleichheit fördernden Funktion der Märkte als Triebfeder ökonomischen Wachstums und gesellschaftlicher Wohlstandsproduktion wird zur „Formel 1“ der neuen Sozialdemokratie. Gerechtigkeit soll nicht länger mit einer Reduzierung von Ungleichheit identifiziert werden; „begrenzte Ungleichheiten“ sollen zukünftig ein Mehr an Gerechtigkeit herbeiführen. Demzufolge gelten Ungleichheiten in Einkommen, Vermögen und Lebenschancen, in einer „Welt der größeren gesellschaftlichen Differenzierung, des intensiven wirtschaftlichen Wettbewerbs und der raschen technologischen Innovation" nicht länger als ein Problem. Sie sollen eher als „ein Katalysator (...) für individuelle (und) (...) auch für gesellschaftliche Entfaltungsmöglichkeiten" betrachtet werden - weil sie zu höherer Produktivität und Wirtschaftsleistung führen (Clement 2000). In der Tradition der Sozialdemokratie galt ökonomisches Wachstum allerdings schon immer als das Allheilmittel, mit dem sich das Problem der Verteilungsgerechtigkeit bearbeiten und zugleich harte Umverteilungskämpfe umgehen ließen. So gesehen: nichts Neues unter dem Mond. Unbeschadet aller ökologisch motivierten Kritik an den „Grenzen des Wachstums“ (vgl. u. a. Daly 1999) erweist sich das „Wachstumssyndrom“ als zählebig. Wie gehabt geht es um die Erhöhung des Verteilungsvolumens im Zeitverlauf. Statt politischer Ergriffe in die funktionelle Wohlstandsverteilung zugunsten wirtschaftlich schwächerer Akteure sollen die Marktkräfte für Wachstum und Innovation sorgen - und dadurch zu „Wohlstand für alle“ führen.

Schnörkelloser und daher unmißverständlicher als die „neuen“ Liberalen in der Sozialdemokratie - und bei den deutschen Bündnisgrünen, in deren Bundestagsfraktion sie bereits die Mehrheit stellen - bringen dies Marktideologen auf den Punkt. Neuerdings ist es den Kämpfern für die freie Marktwirtschaft möglich, nicht nur in dezidiert wirtschaftsfreundlichen Medien sondern auch in gewerkschaftlichen Publikationsorganen (in den Gewerkschaftlichen Monatsheften und in der Mitbestimmung) ihren ,grundsätzlich erfrischenden, neoliberalen Kreuzzug gegen den Korporatismus" zu Ende zu führen; so das Urteil von Wolfgang Streeck (2000), Direktor des Max-PlanckInstituts für Gesellschaftsforschung, über den Wirtschaftsjournalisten Rainer Hank. Im Namen der individuellen Freiheit und eines globalen Hyperwettbewerbs, der auf die Ungleichheit als seinen „sportlichen Motor“ angewiesen ist, fordert Hank (2000) ein „Ende der Gleichheit“. Damit sind freilich nicht die Rechtsgleichheit, die Gleichheit vor dem Gesetz und die Gleichheit aller Menschen im Sinne der „ersten Generation“ der Menschenrechte gemeint. Die These lautet vielmehr, daß der Egalitarismus einer verteilungsgerechten Gesellschaft auf Kosten ihrer Effizienz gehe und die bereits realisierte Gleichheit sich als Blockade der ökonomischen Dynamik auswirke. Die gebetsmüh- 
lenhaft vorgetragene Formel, die sich nun auch die Sozialdemokratie zu eigen macht, lautet: „Wer soziale Gerechtigkeit will, muß Ungleichheit zulassen, weil er damit Wachstum fördert" (ebd.).

\section{Die Chimäre einer wachstumshinderlichen "egalitären Gesellschaft"}

Nun entspricht die deutsche Gegenwartsgesellschaft weder hinsichtlich der Wohlstandsverteilung noch was die Ungleichheit zwischen den Geschlechtern oder jene der Bildungszugänge anbelangt, dem Bild einer egalitären Gesellschaft. Um nur einige der wichtigsten Indikatoren in Erinnerung zu rufen:

Erstens: In Westdeutschland sind die Realeinkommen rückläufig; in ganz Deutschland ist die Verteilung der Markteinkommen seit Beginn der 90er Jahre deutlich ungleicher geworden (vgl. DIW 19/2000). Zugleich hat sich die Schere zwischen den Haushaltseinkommen von Selbständigen und abhängig Beschäftigten resp. Beziehern von Transfereinkommen in den letzten 20 Jahren wieder geweitet. Dies ist in Deutschland (wie in anderen OECDLändern auch) vor allem darauf zurück zu führen, daß die Gewinn- und Vermögenseinkommen sehr viel stärker gestiegen sind als die Einkommen, die durch Arbeitsleistungen von Erwerbstätigen entstanden sind. Dieser Verteilungsprozeß ist durch politische Interventionen verstärkt zu Lasten der Lohnabhängigen beeinflußt worden - und nichts spricht dafür, daß die von der rot-grünen Bundesregierung beschlossene Steuerreform oder gar ihre Rentenreform an dieser Schieflage etwas ändern wird (vgl. dazu die Beiträge von Reuter und Lohauß in diesem Heft).

Zweitens: Trotz aller Rhetorik vom „gender mainstreaming“, das seit 1996 zum offiziellen Prinzip für die Durchsetzung der Chancengleichheit von Frauen und Männern in der EU erklärt wurde, fehlt es nach wie vor an ernsthaften Versuchen, die geschlechtsspezifischen Strukturen von Verteilung und die Ungerechtigkeiten zu korrigieren, die mit den ungleichen Chancen auf Anerkennung und Selbstachtung für Männer und Frauen verbunden sind. Für Frauen gibt es nach wie vor ein „Arbeitsmarktrisiko Kind“ und von einer „partizipatorischen Gleichheit“ (Frazer 1998) hinsichtlich des sozialen Status der Geschlechter sind wir weit entfernt.

Drittens: Obwohl es in den vergangenen dreißig Jahren zu einer Bildungsexpansion gekommen ist, die sehr viel mehr Menschen als früher (insbesondere aber sehr viel mehr Mädchen und Frauen) zu höherer Bildung verholfen hat, bleibt die soziale Herkunft und v. a. die ethnische Zugehörigkeit die entscheidende Determinante beim Erwerb von allgemeiner Bildung und beim Zugang zu beruflicher Ausbildung. Einmal ganz abgesehen von der immer wichtiger werdenden beruflichen Weiterbildung, die mehr und mehr in die (finanzielle) Verantwortung des Einzelnen gerückt wurde und vor allem deswegen als Ver- 
stärker sozialer Ungleichheiten wirkt, weil sie innerhalb der Unternehmen nach dem Matthäus-Prinzip funktioniert: „Wer hat, dem wird gegeben“.

Selbst unter den „Modernisierern“ ist unstrittig, daß es soziale Ungleichheiten wie die genannten gibt. Nur werden diese, wenn sie (auf welchen Umfang auch immer) „begrenzt“ bleiben und (gemessen an welchen Maßstäben auch immer) „vertretbar“ sind, eben nicht länger als ein Problem behandelt (Clement 2000). Auf „dem erreichten Niveau des allgemeinen Wohlstands“ sei ein Anstieg der gesellschaftlichen Ungleichheit nicht nur „unvermeidlich“, sondern auch „grundsätzlich hinnehmbar“ und zur weiteren Erhöhung der wirtschaftlichen Leistungsfähigkeit auch „notwendig“, so noch einmal Wolfgang Streeck, ganz „ohne nervenschonende, didaktische Umschweife“, in einer Lobeshymne auf die „zivile Bürgergesellschaft“, jene neue, von Gerhard Schröder lancierte politische „Formel für die deutsche Sozialdemokratie“, mit der endlich Abschied genommen werden soll, von deren „staatszentrierten Irrealsozialismus der westdeutschen Wohlstandsperiode“ (Streeck 2000: 28f). Nicht zuletzt an die begriffsstutzige "Sozialarbeiterfraktion in Partei und Gewerkschaften“ richtet sich die Botschaft, daß vom Staat „nicht mehr alles, sondern nur noch immer weniger" erwartet werden darf; „die Gesellschaft und der Einzelne müssen und können mehr Verantwortung für sich selbst übernehmen, also mehr aus eigener Tasche bezahlen (....); der Staat als Vollkaskoversicherung hat ausgedient" (ebd.: 28).

Zeitgemäß wäre demnach ein Gerechtigkeitsverständnis, das erstens eine weitere Verschiebung der Verteilungsrelationen zugunsten des Faktors Kapital, d.h. der Zinsen und Profite und auf Kosten des Faktors Arbeit, also der Löhne und Gehälter legitimiert und zweitens die Reduzierung sozialer Sicherheit auf ein Minimum zuläßt. Die Beziehungen zwischen Individuum, Gesellschaft und Staat werden so reformuliert, daß bestenfalls mit Hilfe bei der Bewältigung von Leistungszumutungen des Marktes gerechnet werden kann. Einrichtungen des sozialstaatlichen Schutzes vor den Wirkungskräften der durch weitreichende Deregulierungsmaßnahmen „befreiten“ Märkte erhalten die Funktion eines „Lazarettwagens“ (Scharping 2000); dieser wird nur dann in Bewegung zu setzen sein, wenn das Individuum und danach auch noch die „Zivilgesellschaft“ versagt haben: „Ein sozialdemokratisches Grundsatzprogramm denkt vom einzelnen Menschen, denkt vom Individuum, denkt von seinen Rechten und Möglichkeiten her, von der Entfaltung seiner Freiheit und stellt danach die Frage, was die Individuen in ihren freien Zusammenschlüssen selbst leisten können, wozu also die Zivilgesellschaft fähig ist. Und dann stellt sie die Frage nach den staatlichen Regulativen ...", so der Vorsitzende der Programmkommission der SPD Rudolf Scharping (ebd.).

Weil Eingriffe der Politik in die Funktionsweise der Marktkräfte auf dem „Dritten Wege“ nicht vorgesehen sind, ist es nicht ganz abwegig, daß „moderne Sozialdemokraten“ sich auf eine „Hierarchie von Gerechtigkeitszielen“ 
verständigen könnten, wie sie von liberalen „Vordenkern“ in die Debatte gebracht werden (vgl. Merkel 2000). Die „Teilhabe“ am Bildungssystem stünde dabei ganz oben auf der Prioritätenliste; was indes keineswegs bedeuten müßte, daß Bildung, so wie bisher, in öffentlicher Regie und kostenlos angeboten werden müßte. Schließlich hat der Ruf nach „Elitenförderung“ und „mehr Wettbewerb" längst seinen konservativen Beiklang verloren. Auch stört sich offensichtlich kaum jemand an der zumindest widersprüchlichen Empfehlung, die soziale Malaise zunehmender sozialer Ungleichheit durch individuelle Höherqualifizierung zu kurieren. Letztlich geht es vor allem darum, Bildung als einen anderen Weg der Integration in den Arbeitsmarkt zu propagieren, der, wie die um jeden Preis erzwungene Beteiligung am Arbeitsmarkt, die auch vor einer „Absenkung dafür hinderlicher sozialer Sicherungsstandards" nicht zurück schreckt (ebd.: 31), eine sekundäre Verteilung überflüssig macht. Gerecht wäre demnach drittens wohl auch eine Verhinderung von Armut und zwar dadurch, daß die Gewährung von Transfereinkommen an eine Verschärfung der Pflichten zur Wiederaufnahme von Arbeit geknüpft wird. „Ein Sozialstaat, der über Grundeinkommen, großzügige Sozialhilfen, locker definierte Zumutbarkeitskriterien die Nichtaufnahme von Erwerbsarbeit ermöglicht oder dazu anreizt und durch vermeintlich soziale Regulierungen den Arbeitsmarkt gegenüber outsidern, den Frauen und Jugendlichen abriegelt, ist aus dieser Perspektive sozial ungerecht“ (ebd.: 31f). Gegenüber diesen drei „Gerechtigkeitszielen“ erweist sich die Verteilungsrechtigkeit im Sinne einer Verringerung der Einkommens- und Vermögensspreizung als ein nachrangiges, um nicht zu sagen kontraproduktives Ziel, wird doch erwart, daß eine noch größere Einkommensungleichheit zu höherer Produktivität und Wirtschaftsleistung führt.

Die Grundidee einer Funktionaliät von sozialer Ungleichheit ist alles andere als neu. Auch die Instrumente sind es nicht, mit denen die heute als störend und belastend empfundene Verteilungsgerechtigkeit in den Hintergrund gedrängt wird. Immer wieder geht es um Steuersenkungen, eine stärkere Lohnspreizung und die (weitere) Flexibilisierung des Arbeitsmarktes. All dies sind aber Maßnahmen, die in Deutschland (wie anderswo auch) bereits seit Beginn der 80er Jahre verstärkt zum Einsatz kommen. Bis heute haben sie nicht $\mathrm{zu}$ einem beschleunigten Wachstum und vor allem nicht zu einer Ausweitung von existenzsichernden Beschäftigungsverhältnissen geführt ${ }^{1}$. Daß die

1 Bekanntermaßen werden die Gewinne der Unternehmen, die durch die genannten Maßnahmen während der letzten Dekade deutlich gestiegen sind, nicht in die Realökonomie, also zur Ausweitung von Produktion und Beschäftigung investiert, sondern auf die Finanzmärkte geschafft. Das ist auch nicht verwunderlich, solange die realen Zinsraten auf Finanzvermögen, die auf globalisierten und deregulierten Finanzmärkten gebildet werden, oberhalb der durchschnittlichen Zuwachsraten des realen Wachstums liegen - und dies ist nicht nur in Deutschland sondern auch in den anderen Industrieländern seit Beginn der 80er Jahre der Fall (vgl. Altvater 2000). Unternehmer werden ihre Gewinne nur dann reinvestieren oder zur 
„Modernisierer“ auf ökonomisches Wachstum und technischen Fortschritt als notwendige Voraussetzung für die Lösung all unsere Probleme: für die Sanierung der Staatsfinanzen, die Beseitigung der strukturellen Massenarbeitslosigkeit, für die Finanzierung der Alterssicherung in einer alternden Gesellschaft und für die Stabilisierung unserer sozialen Sicherungssysteme in Zeiten globalen Wettbewerbs (Müller-Pantenberg 1998) setzen, ist ebenso wenig eine neue Strategie. Neu ist allerdings, daß die Schimäre einer (wachstumshinderlichen) egalitären Gesellschaft herhalten muß, um ein Mehr an Ungleichheit zu rechtfertigen, dessen ökonomischer Sinn zweifelhaft ist und dessen sozialen Folgen für die Demokratie in Deutschland erhebliche Gefahren mit sich bringen. Die mentale Kolonialisierung der Sozialdemokratie durch den Neoliberalismus, welche darin zum Ausdruck kommt, steht jedenfalls in scharfem Gegensatz zu ihrem historischen Projekt.

\section{Verteilungsgerechtigkeit in Zeiten der Globalisierung}

Im Zentrum der aktuellen Gerechtigkeitsdebatte steht das Verhältnis von Markt und Politik. Was können wir den Märkten überlassen und was bleibt im Hinblick auf die Herstellung von Gerechtigkeit Sache der Politik? Im Verständnis von Adam Smith galten Marktergebnisse deswegen als gerecht, weil alle Marktakteure die gleichen Freiheiten genießen. Allerdings hatte der Klassiker der Politischen Ökonomie (ebenso wie David Ricardo) dabei Warenund Gütermärkte im Blick, nicht aber globalisierte und deregulierte Finanzmärkte. Diese sind aus strukturellen Gründen zur Herstellung sozialer Gerechtigkeit ungeeignet: Sie erzeugen Gläubiger- und Schuldner Verhältnisse, die zerstörerische Wirkungen auf den gesellschaftlichen Zusammenhalt ausüben. Die gesellschaftliche Arbeit mag ein unverzichtbares Element gesellschaftlicher Kohäsion sein, das Geld aber ist unbedingt ein Mittel der Gesellschaftsspaltung. Davon war bereits Aristoteles, der nichts von den verheerenden Auswirkungen globaler Währungs- und Finanzkrisen wissen konnte, überzeugt. Da unter normalen Umständen in absehbarer Frist aus Schuldnern keine Gläubiger werden und umgekehrt, kommt es infolge der einseitigen Verschuldung zu einem höchst ungerechten Reichtumstransfer zugunsten von Geldvermögensbesitzern. Das läßt sich seit Ausbruch der Schuldenkrise in den 80er Jahren in den Ländern des Südens und an den ökonomischen und sozialen Folgen der Wirtschafts- und Finanzkrisen in den 90er Jahren in Mexiko, Asien, Rußland und Brasilien - exemplarisch studieren.

Ausweitung ihrer Geschäftstätigkeit Kredite auf dem Finanzmarkt aufnehmen, wenn sie mit einer Rendite rechnen können, die dem Niveau der globalen Zinsraten entspricht. Oder anders formuliert: Wer reales Wachstum stimulieren will, muß zuerst Druck auf eine Senkung der viel zu hohen Realzinsen ausüben. Dies hat bekanntlich Oskar Lafontaine versucht, wir wissen alle mit welchem Ergebnis. 
Doch nicht allein unter dem Gesichtspunkt der „globalen Gerechtigkeit“ erweist sich das Vertrauen in die wohlstandsschaffende Funktion von Märkten als naiv, wenn nicht gar als zynisch (vgl. dazu auch O'Neill 1998). Jenes „realistische Mehr an Gerechtigkeit“, das in Zusammenhang mit „begrenzten Ungleichheiten“ vom Wirken der Marktkräfte erwartet wird (Clement 2000), muß noch aus einem anderen Grunde enttäuscht werden: Anders als in der Gerechtigkeitstheorie von John Rawls unterstellt, haben wir es heute eben nicht mit weitgehend autarken Nationalstaaten zu tun, mit mehr oder weniger abgeschlossenen Vereinigungen von Menschen, die für ihre gegenseitigen Beziehungen gewisse Verhaltensregeln als bindend annehmen und einen gemeinsamen Maßstab anerkennen, nach denen ihre jeweiligen Ansprüche zu beurteilen sind. Anders auch als zu Zeiten von Adam Smith haben wir es heute nicht nur mit den für die lokalen Gesellschaften unkalkulierbaren und manchmal verheerenden Wirkungen deregulierter Finanzmärkte zu tun, auch die noch immer national regulierten Arbeitsmärkte geraten zunehmend unter Globalisierungsdruck. Diese Entwicklung hat weitreichende Konsequenzen sowohl für die Leistungsgerechtigkeit verbunden, also für jenes liberale Element der Verteilungsgerechtigkeit, das für eine Mehrheit der deutschen Bevölkerung, insbesondere für die „soziale Mitte“ im sozialstrukturellen Sinne (vgl. Vester 1999) noch immer eine ganz große Rolle spielt, als auch für Prinzip der Bedarfsgerechtigkeit.

\section{Erosion der Leistungsgerechtigkeit}

Die Erosion des Leistungsprinzips wird von den liberalen Verfechtern marktgesteuerter Formen von „Gerechtigkeit“ vornehm verschwiegen. In der Vergangenheit wurden anerkannte Maßstäbe für Leistungsgerechtigkeit im nationalen Rahmen ausgehandelt und in nationalspezifischen Tarifvertragssystemen kodifiziert. Normen, Regeln und Gesetze, für die der Volkssouverän verantwortlich war, bildeten die Grundlage für ein Bezugssystem, das Rechte und Pflichten, Lasten und Entschädigungen, Leistungen und Gegenleistungen auf die zielgerichtete Tätigkeit und deren dominante Form: die betrieblich organisierte, „normale“ Lohnarbeit gründete. Als leistungsgerecht galten solche Standards, die sich auf eine meßbare Arbeitsleistung und auf erworbene Qualifikationen bezogen. In der Regel handelte es sich dabei um Qualifikationen, die in formalisierten Bildungsprozessen erworben wurden und die dank staatlicher Garantien der Abschlüsse, also durch die öffentlich-rechtliche Zertifizierung der Qualifikationen ein gewisses $\mathrm{Maß}$ an Sicherheit im Lebensund Erwerbsverlauf ermöglichten. Allerdings hatte die auf diese Weise bewirkte Leistungsgerechtigkeit stets exkludente Züge; sie schloß die vornehmlich von Frauen verrichteten reproduktiven Tätigkeiten ebenso aus wie „atypische Beschäftigungsformen“, die im "goldenen Zeitalter des Kapitalismus“ 
zwischen 1950 und Mitte der 70er Jahre allerdings nur eine marginale Rolle spielten. Mit der Beseitigung von Konkurrenzgrenzen im Prozeß der ökonomischen Globalisierung - genauer: durch die Öffnung von Märkten und durch die Wirkungen neuer Informations- und Kommunikationstechnologien - verlieren das „fordistische Normalarbeitsverhältnis“ und nationale Systeme der industriellen Beziehungen ihre normprägende Bedeutung. Zugleich werden sowohl für den Zugang zum Arbeitsmarkt wie für die Bezahlung erbrachter Arbeitsleistungen individuelle Eigenschaften (berufliche und geographische Mobilität, zeitliche Verfügbarkeit, hohe Belastbarkeit, eine generalisierte Leistungsbereitschaft, die Fügsamkeit gegenüber jedweden Anforderungen sowie kommunikative und reflexive Kompetenzen) gegenüber den erworbenen (und durch staatliche Zertifikate garantierten) Qualifikationen immer wichtiger (vgl. Mahnkopf 1999b).

Alle diese Entwicklungen tragen dazu bei, daß der Maßstab für durchschnittliche Leistungen und durchschnittliche Leistungsansprüche schwindet, im nationalen Rahmen ebenso wie auf der Ebene einer Branche oder innerhalb eines Unternehmens. In der Blütezeit der fordistischen Massenproduktion war ein solches einheitliches Maß für wirtschaftliche Leistungsansprüche die Basis jener gewerkschaftlichen Lohnpolitik, die Belegschaften mit einer starken Verhandlungsposition in einen Solidarverband eingliederte. Dadurch erhielten Arbeitnehmer mit einer schwachen Marktmacht mehr als sie aufgrund ihrer Marktposition hätten durchsetzen können. Doch unter den Bedingungen globalen Wettbewerbs werden die Standards - bei Preisen, Kosten, Qualität, Technik, Design und nicht zuletzt bei der Rendite - im abstrakten Raum einer Geoökonomie gesetzt. Bislang gültige Normen, seien dies Leistungs- oder Verteilungsnormen, die mit diesen Standards, insbesondere mit dem alles überwölbenden „shareholder value“ kollidieren, werden außer Kraft gesetzt durch Maßnahmen der Deregulierung, Liberalisierung und Flexibilisierung oder sie werden in einem Prozeß der „Informalisierung“ von Arbeits- (und Geld-) Beziehungen schlichtweg unterlaufen.

Die mit der Globalisierung aufs engste verbundene Tertiarisierung von Ökonomie und Beschäftigung ist hier von entscheidender Bedeutung: Mit der Verbreitung von wissensgestützten Dienstleistungsökonomien wird der Wert einer Leistung vornehmlich über Angebot und Nachfrage auf globalen Märkten bestimmt; auf die Bedeutung der einzelnen Leistungen für den gesellschaftlichen Lebenszusammenhang (die entsprechend proportional abgegolten werden könnte) kommt es dabei immer weniger an. Vor allem aber läßt sich der Umfang der „Produktivkraft Wissen“ oder die Fähigkeit, diese zu erweitern und anzuwenden, an keinem einheitlichen Maßstab messen. Weil die meisten Arbeiten, die routinemäßig genug sind, um gemessen werden zu können, auch an technische Systeme übertragbar sind, wird die Wertschöpfung heute vor allem dort lokalisiert, wo es um die Bewältigung von Aus- 
nahmesituationen geht. Insbesondere die Informationsökonomie scheint jeglichen Bezug zum Arbeitszeitaufwand als dem Maßstab, nach denen Normen und Regeln des Tausches abgegolten werden, verloren zu haben². Vor allem in der „new economy“ sind Geschick, Orginalität und die Schnelligkeit, mit der Probleme identifiziert und gelöst werden, mit Erfolgsprämien belegt; die Bewältigung von Standardabläufen hingegen, egal wie anstrengend, belastend oder anspruchsvoll diese auch sein mögen, unterliegen einem Entwertungsprozeß (vgl. dazu Altvater/Mahnkopf 2000).

Mit dem Bedeutungsverlust, den das quantitative Maß der Arbeitszeit als $\mathrm{Maß}$ der Leistungsgerechtigkeit erfährt, sind einige weitreichende Konsequenzen verbunden: Erstens handelt es sich bei den auf globalen Märkten gebildeten Standards um etwas eher Fluides. Anders als die Regeln und Normen, die im nationalen Rahmen (in demokratischem Prozedere) gebildet wurden und über Konjunkturen und Krisen hinweg zu etwas relativ Festem und Kohärentem wurden - beispielsweise zu einem „Modell Deutschland“ mit all seinen Facetten - verändern sich die Standards (und manchmal selbst die Regeln des Wettbewerbs) sehr schnell. Daher gibt es keinerlei Sicherheit mehr, daß „Leistung sich lohnt“. Nach den Spielregeln des „Rattenrennens“ werden nicht nur die Unbeweglichen, Leistungsschwachen und Langsamen zu Verlierern, sondern auch viele von denen, die lange Zeit mit einigem Erfolg versucht haben, die „benchmarks“ des Weltmarktes zu erfüllen. Denn irgendwo auf der Welt waren andere, von der „neuen Ökonomie der Zeit“ gehetzte Menschen noch flexibler, besser und schneller als sie. So gesehen verbürgt gerade die Erbringung von quantifizierbaren Leistungen im Gegensatz zu früher keine längerfristigen Sicherheiten mehr, weder im Hinblick auf Karriereverläufe noch hinsichtlich der individuellen Lebensplanung.

Zweitens ähneln immer mehr Märkte denen des Spitzensports und des Kunstgeschäfts. Schon kleinste Leistungsdifferenzen schlagen sich in riesigen Ertragsdifferenzen nieder. Die Gewinner (und vielleicht noch einige der Nächstplazierten) bekommen alles, das weite Feld der Wettbewerber aber geht leer aus. Wo es nur noch einige wenige Gewinner und viele Verlierer gibt, kann einer für durchschnittliche Leistungen nicht viel erwarten. Nur so ist $\mathrm{zu}$ verstehen, daß inzwischen nicht nur IT- und Softwarefirmen nach erleichterten Arbeitsbedingungen für ausländische Spezialisten verlangen, sondern auch die mittelständisch geprägte deutsche Elektroindustrie in einen

2 Auf einen wichtigen Nebeneffekt dieser Entwicklung verweist André Gorz: „,...es wird praktisch unmöglich zu bestimmen, wo die Arbeit anfängt und wo sie aufhört, was zu ihr gehört und was zur Nicht-Arbeitszeit. Denn die Zeit, die wir brauchen, um Wissen und Kenntnisse zu erwerben und zu erweitern, unsere Fähigkeiten möglichst allseitig zu entfalten, diese Zeit könnte als 'mittelbare Arbeit' gelten, da sie ja für die Produktivität der unmittelbaren Arbeit ausschlaggebend ist und sogenanntes 'menschliches Kapital', Wissenskapital, das mit fixem Kapital gleichgesetzt sein kann, herstellt“ (Gorz 2000: 610). 
„Kampf um die besten Köpfe weltweit“ eingetreten ist. Die Über- und die Unterbewertung von Leistungen ist gleichsam konstitutiv für eine „wissensgestützte“ Dienstleistungsökonomie und dies hat viel mit der unterschiedlichen Mobilität von „Produktionsfaktoren“ zu tun. Während früher, Zygmunt Bauman (1999) nennt diese Zeit die „schwere Moderne“, Kapital und Arbeit verhältnismäßig fest an einen Ort gebunden waren und daher der Betrieb als der „kardinale Ort“ (Th. Geiger) betrachtet wurde, an dem die gegensätzlichen Interessen von Kapital und Arbeit aufeinander trafen - ein „umkämpftes Terrain (R. Edwards) zwar, doch auch eine Ära von längerfristigen Bindungen und Bündnissen - sind heute, in der „leichten Moderne“, einige Akteure ungebunden wie nie zuvor. Die Macht ist ganz ungeteilt auf der Seite derer, denen es gelingt, Handlungen unvorhersehbar zu gestalten und die Handlungen der Gegenspieler, zumal solcher, die ihren Ort nicht verlassen können, normativ zu regulieren (vgl. Bauman 1999 sowie Attali 1999). Für die „mobilen Produktionsfaktoren“, die Besitzer von Sach- und Geldkapital und die hochqualifizierten Arbeitskräfte (insbesondere die sogenannten „high potentials“ in den produktionsnahen Dienstleistungsbereichen der „new economy") hat dies die angenehme Folge, daß sie ein Mehrfaches der einstmals üblichen Spitzenverdienste für sogenannten „Leistungsträger“ einstreichen können. Gleichzeitig werden die Leistungen der weniger mobilen Arbeitskräfte - seien diese nun abhängig Beschäftigte oder kleine Gewerbetreibende, welche die globalen Standards nicht erfüllen - zu deutlich schlechteren Konditionen nachgefragt und dies, obwohl sie im Vergleich zu früher schneller, flexibler, billiger, kundenfreundlicher geworden sind.

Dabei kommt es faktisch zu einer Entkoppelung von Aufwand und Ertrag, von Leistung und Einkommen. Wenn ein immer größerer Teil der Einkommen aus Optionsscheinen auf Aktien von Unternehmen bestehen, wird das Einkommen abhängig von der Entwicklung der Börsenkurse. Nicht die individuelle Leistung sondern das „Prinzip Zufall“ entscheidet letztlich über Zeitpunkt und Umfang einer „Erfolgs“-Prämie (vgl. auch Neckel 2000). Wenn diese Entwicklung sehr zutreffend als „Amerikanisierung“ bezeichnet wird, so nicht allein deshalb, weil sich mit der Erosion des Leistungsprinzips als öffentlich rechtfertigungsfähiger Maßstab für Unterschiede von Vermögen, Status und Macht die Einkommen deutscher Topmanager an die ihrer US-amerikanischen Counterparts annähern ${ }^{3}$, sondern auch deshalb, weil damit ein spezifisch europäischer Gerechtigkeitsdiskurs abgebrochen wird: Ei-

3 Nach Berechnungen von „headhuntern“, die seit 1997 für „DIE ZEIT“ durchgeführt werden, sind die Einkommen deutscher Topmanager in Unternehmen, die im Deutschen Aktienindex (Dax) gelistet werden, in nur zwei Jahren immerhin um 40\% gestiegen, auf durchschnittlich 2,5 Millionen Mark im Jahr 1999. Im Kreditwesen oder bei DaimlerChrysler realisieren Vorstandsmitglieder inzwischen aber durchaus „Weltmarktpreise“ von über 6 Millionen Mark und mehr (DIE ZEIT vom 29.Juni 2000). 
nerseits galten Unterschiede, die durch Arbeit bedingt sind, in der europäischen Tradition stets als legitimer denn Unterschiede, die durch Geburt, Eroberung oder Zufall zustande gekommen sind. Andererseits ging es im europäischen Modernisierungsprozeß seit der Industriellen Revolution immer darum, einen Ausgleich, eine Mindestbalance zu finden - zwischen dem Anteil des gesellschaftlichen Reichtums, den man durch Arbeit erwerben kann und dem, der durch Geld erreichbar ist.

Drittens schließlich kommt es in Folge der beschriebenen Entwicklungen zu einer Verschiebung der Verteilungskonflikte von der vertikalen zur horizontalen Ebene: Unter den Bedingungen von Währungskonkurrenz zwischen regionalen Wirtschaftsblöcken und Steuerkonkurrenz zwischen einzelnen Nationalstaaten unterläßt die staatliche Politik alles, was die Wettbewerbsfähigkeit mobiler Produktionsfaktoren beeinträchtigen könnte. Kategorisch hat der Präsident des Kieler Instituts für Weltwirtschaft Horst Siebert jüngst festgestellt:

„Es geht nicht mehr nur allein um den Wettbewerb auf den Gütermärkten, sondern auch um den Wettbewerb auf den Faktormärkten und zwar um die mobilen Produktionsfaktoren, um das mobile Kapital, das mobile technische Wissen und die hoch qualifizierten Arbeitskräfte. In diesem Wettbewerb der Staaten handelt es sich darum, mit den Instrumenten Steuer, Infrastruktur und institutionellen Regelungen, Kapital, technologisches Wissen und qualifizierte Arbeitskräfte im Land zu halten und ins Land zu holen" (Siebert 2000).

„Verfehlt“ wäre es auf jeden Fall, „über Verteilung zu diskutieren“ (ebd.), denn dies könnte die begehrten, mobilen Produktionsfaktoren verschrecken. Mit anderen Worten: Die Dynamik des technisch-ökonomischen Strukturwandels führt zu einer sinkenden Nachfrage nach gering qualifizierten Arbeitskräften und zu einer steigenden Nachfrage nach „Bestleistungen“; gleichzeitig ist diese Dynamik dafür verantwortlich, daß sich der Zusammenhang von Aufwand und Ertrag des sozial gebildeten („Human“-)Kapitals lockert. Denn es geht nur noch darum, Wissensressourcen, wo auch immer sie sich befinden mögen, zu erschließen, nicht aber darum, sie im Lande selbst zu erzeugen. Unter diesen Bedingungen wird der Verzicht auf eine Umverteilung zu Lasten aller mobilen Faktoren (also nicht nur des Sach- und Geldkapitals sondern auch der „Besitzer von Humankapital“) zu einem notwendigen Element von „gutem Regieren“.

So wird es den Gewinnern der Globalisierung leicht gemacht, Ort und Höhe der Besteuerung ihrer Einkommen selbst festzulegen. Für den immobilen Teil der wirtschaftlichen Akteure, die in der Reichweite der nationalen Umverteilungssysteme bleiben, aber bedeutet dies, daß sie soziale Leistungen welcher Art auch immer zunehmend aus ihrem eigenen Einkommen finanzieren müssen. Der nationale „Reichtumskreislauf“" zerbricht und der Verteilungskonflikt nimmt immer stärker den Charakter eines drastischen Nullsummenspiels an. Statt auf der bislang zentralen vertikalen Achse von Arbeit und Kapital, von 
„Oben und Unten“ in den Grenzen eines Nationalstaats und einer „Volks“wirtschaft konkurrieren nun ,aneinander grenzende Territorien, Norden gegen Süden, Nord-Westen gegen Nord-Osten, Junge gegen Alte, Erwerbslose gegen Rentner, Fabrikgemeinschaften gegen andere Fabrikgemeinschaften, Beschäftigte des Dritten Sektors gegen öffentliche Angestellte im Dienstleistungssektor, abgesicherte Arbeiter gegen prekäre Arbeiter, Familien- und Nachbarschaftsgruppen gegen benachbarte Familien- und Nachbarschaftsgruppen (Revelli 1999: 119). Ein Gerechtigkeitsdiskurs, der dieser Verschiebung mitmacht und sie zu legitimieren trachtet, mag partikuare Interessen bedienen, kann aber nicht vergessen machen, daß der (legitimationsstiftende) Zusammenhang von Leistung und Belohnung zerreißt. Dieser Riß wird als eine „Gerechtigkeitslücke“ erfahren, die sich durch „sozial-liberale“ Wettbewerbsrhetorik nicht lange verkleistern läßt.

\section{Abschied von der Bedarfsgerechtigkeit}

Doch nicht nur die Norm der Leistungsgerechtigkeit, die noch immer eine hohe Legitimations- und Integrationskraft hat, ist einem Erosionsprozeß ausgesetzt. Auch das auf die Schwachen und Armen der Gesellschaft bezogene Prinzip der Bedarfsgerechtigkeit steht zur Disposition. Viele Menschen, deren Leistungen auf dem Arbeitsmarkt nicht mehr nachgefragt werden oder die nur einfache Leistungen anbieten können - gering qualifizierte, ältere und gesundheitlich beeinträchtigte Menschen vor allem - werden zu einer „überflüssigen Restbevölkerung“. Das „neue Subproletariat“ aus Arbeitslosen, Überforderten, unbeschäftigten Frauen, schlecht ausgebildeten Jugendlichen kann mit Umverteilungsmaßnahmen zu seinem Gunsten nicht mehr rechnen, weil, so Peter Glotz (2000) in einer „beschleunigten Gesellschaft“, die Gewinner „nicht mehr teilen wollen“. Das neue Subproletariat muß allerdings durch „Brot und Spiele“ bei Laune gehalten werden, damit es sich nicht von - so Glotz weiter - „tragik/komischen“, „zivilisationsängstigen“, „,antirationalen Antikapitalisten“, „Entschleunigern und Ökologen“ in neue „Kulturkämpfe“ führen läßt.

Dies ist auch der Grund, weshalb die Vordenker und Propagandisten des „Dritten Weges“ sozialen Fortschritt nicht mehr mit einer Ausdehnung von sozialen Rechten gleichsetzen wollen, sondern für eine enge Kopplung von Rechten und Pflichten plädieren. Auch soll sozialer Fortschritt nicht mehr am Zuwachs von öffentlichen Gütern (beispielsweise an einer verbesserten und öffentlich subventionierten Kinder- und Altenbetreuung) ablesbar sein. Die „befreiende Individualisierung des Marktes“ (Streeck 2000) soll anstelle jener Gleichheit treten, die eine staatliche Grundversorgung auf hohem Niveau allen Bürgern gewährleistet. Denn als Konsumbürger hätten sich die Bürger/innen der Bundesrepublik ja ohnehin „daran gewöhnt (...), individuell 
bedient statt einheitsversorgt zu werden - also Spaßbäder statt Stadtbäder zu benutzen" (ebd.: 28). Daher gilt Gleichheit nicht länger als ein Wert, der um seiner selbst willen anzustreben wäre. Nur so weit Ungleichheit eine Bedrohung für die gesellschaftliche Ordnung und vor allem für die ökonomische und technologische Entwicklung der Gesellschaft darstellt, muß Politik auf ihren Abbau zielen. Indem das Konzept der Gleichheit durch das der „Inklusion“ ersetzt wird (vgl. dazu insbesondere Giddens 1999a, 1999b), verengt sich der Sinn von Gleichheit auf seine soziale Nützlichkeit: Die „Teilhabe“ so lautet die Version von „Inklusion“ im deutschen Diskurs über „Gerechtigkeit“ und „Zivilgesellschaft“ - an Bildung und Arbeit befördert die Integration der die Gesellschaft und zugleich die wirtschaftliche und technische Entwicklung. „Nicht-Teilhabe“ an (oder „Exklusion“ aus) den Strukturen der Arbeitsgesellschaft gilt hingegen als eine Störung, die durch „modernes Regieren" beseitigt werden kann. Die ethische Begründung des Gleichheitsgrundsatzes ergibt sich somit aus den Funktionsbedingungen der gegenwärtigen Gesellschaft selbst und Ungleichheit, die als Nicht-Teilhabe virulent wird, scheint mit Strukturprinzipien der im Kern für unantastbar gehaltenen Gesellschaftsordnung nichts zu tun zu haben.

Wo früher Sozialpolitik darauf abzielte, für alle Bürger Sicherheit der Einkommen und der Lebensumstände zu gewährleisten, soll nun die soziale Nützlichkeit (oder die „employability“) den Ausschlag dafür geben, ob eine Hilfeleistung gewährt wird. Für ein solches Konzept von Sozialpolitik, das an den „residualen Wohlfahrtsstaat“ US-amerikanischer Prägung erinnert, der nur im Falle von akutem Versagen des Arbeitsmarktes und der Familie einspringt und seine Leistungen einzig jenen Notleidenden zukommen läßt, die Unterstützung „verdienen“, ließe sich in Deutschland womöglich parteiübergreifende Unterstützung organisieren (vgl. dazu auch Lohauß in diesem Heft). Möchte doch auch die CDU einen „fairen Sozialstaat“, der keine Anreize setzt, „die ein Leben auf Kosten der Allgemeinheit attraktiv und erstrebenswert machen“ (CDU-Sozialstaatskommission zit. nach „Frankfurter Rundschau“ vom 21./22. Juni 2000). Keinesfalls aber könnte sich eine zur disziplinierenden Armenpolitik degradierte Sozialpolitik - was unter den Modernisierern jeglicher Couleur aber üblich ist (vgl. Merkel 2000) - auf das „dänische Beispiel“ berufen: Wohl müssen sich in Dänemark Arbeitslose umschulen lassen und zugeteilte Jobs annehmen, um Arbeitslosengeld zu erhalten und fraglos gibt es dort auch nur einen minimalen Kündigungsschutz. Dennoch würde das dänische Beispiel nur dann als „Vorbild“ für eine enge Kopplung von Rechten und Pflichten herhalten können, wenn seine Befürworter nicht gleichzeitig für niedrigere Sozialleistungen als „Anreiz fürs Arbeiten" plädierten. Die Flexibilität des dänischen Arbeitsmarktes wird gerade durch einen sehr hohen Standard der sozialen Sicherung ermöglicht. Insofern steht das „Modell“ dafür, daß unter den Bedingungen vermehrten Jobwech- 
sels das soziale Netz engmaschig und tragfähig gehalten wird.

Wenn es sich bei der Erosion des (liberalen) Prinzips der Leistungsgerechtigkeit um einen marktinduzierten Prozeß handelt, dessen Folgen für die „soziale Mitte“ im neuen Ungleichheitsdiskurs eher verschwiegen, denn öffentlich gerechtfertigt werden, so erfolgt die Suspendierung der Bedarfsgerechtigkeit jenes zweiten, eher der sozialistischen Tradition zuzurechnenden Elements der Verteilungsgerechtigkeit - in aller Offenheit und mit einer unmißverständlichen Botschaft an die Armen und sozial Benachteiligten der Gesellschaft: In Zeiten der Globalisierung ist dem Ziel wirtschaftlicher Effizienz gegenüber allen anderen politischen Zielen, insbesondere dem der Herstellung möglichst gleicher Lebensbedingungen für alle Bürger, Vorrang einzuräumen. Wer seiner Pflicht zur Erwerbstätigkeit nicht nachkommt, muß damit rechnen mit einer Minimalversorgung abgespeist zu werden.

Wie die Erosion des Prinzips der Leistungsgerechtigkeit so ist auch die Aufkündigung der Bedarfsgerechtigkeit folgenreich: Erstens bewirkt eine Strategie der erzwungenen Arbeitsmarktintegration von Armen - egal in welche Arbeitsverhältnisse und egal zu welchem Preis - eine stärkere Differenzierung und Absenkung der Löhne im unteren Lohnbereich (Kombilöhne, Schaffung eines Niedriglohnsektors) sowie eine weitere Prekarisierung von Beschäftigungsverhältnissen (vgl. Schäfer 2000a sowie Zilian in diesem Heft). Wenn jedoch der Staat Niedriglöhne, die bis zu 30\% unter den Tariflöhnen liegen dürfen, flächendeckend mit Steuermitteln subventionieren würde, könnte die materielle Abwertung letztlich alle (noch) tarifierten Arbeitsverhältnisse treffen.

Zweitens hilft die erzwungene Arbeitsmarktintegration, das Leitbild des „flexiblen Menschen" (R. Sennett) als ein alternativloses Menschenbild selbst in den Köpfen von Menschen zu verankern, die dem von außen gesetzten Zwang zur Anpassung an wechselnde Anforderungen nicht einmal die Illusion persönlicher Freiheit abgewinnen können. Dabei wird auch noch suggeriert, es ließe sich dieses „neoliberale Selbstdressurprogramm“ (Strasser 2000b; vgl. zur Kritik ebenso Zilian 2000; Mahnkopf 1999) mit dem zugleich gewünschten „zivilgesellschaftlichen Engagement“ zur Deckung bringen. Doch mit der beständigen Selbstoptimierung des „unternehmerischen Einzelnen“ gehen gerade viele der Fähigkeiten und Kompetenzen (Solidarität, Empathie, Hilfsbereitschaft), die im Zuge einer kompensatorischen Moralisierung der Politik eingeklagt werden, definitiv verloren - wenn sie denn überhaupt ausgebildet werden könnten.

Drittens impliziert eine Kopplung von Transferleistungen an die Bereitschaft, irgendeine Erwerbsarbeit aufzunehmen, daß Tätigkeits- und Lebensformen, die zeitgleich eine rhetorische und teilweise auch einer rechtliche Aufwertung erfahren, faktisch entwertet werden. Dazu rechnen vor allem die Betreuungsund Pflegeleistungen, die von alleinerziehenden und gerade deshalb häufig 
armen und auf Sozialhilfe angewiesenen Frauen ausgeführt werden. Die „Vollkasko-Gesellschaft“ der zu Ende gegangenen Sozialstaatsära, die von den Propagandisten des „Dritten Weges“ als Modernisierungshemmnis gebrandmarkt werden, war eine wesentliche Voraussetzung für eine teilweise Befreiung der Frauen von ihrer traditionellen Pflicht zur „spontanen und individuellen“ Solidarität. Sie durften die „Sozialhilfe von 'Vater' Staat als gerechte Entlohnung ihrer Erziehungsleistungen und als Alternative zur Versorgungsehe" verstehen (Kunz 2000, Herv. BM). Die Bedingungen eines nach angelsächsischem Vorbild gestalteten „workfare“, aber auch die Konturen der absehbaren Rentenreform in Deutschland beschneiden hingegen die materiellen Voraussetzungen für jegliche „Entstandardisierung von Lebensläufen“.

Viertens aber leitet das als „modernisierte Volkspädagogik“ (Ehrke 2000: 18) daher kommende Programm der "Teilhabe durch (jedwede) Erwerbsarbeit“ eine möglicherweise folgenschwere Diskursverschiebung ein. An die Stelle von Rechtsansprüchen auf bestimmte Leistungen, die sich aus dem Menschen- und dem Bürgersein der Individuen herleiten und den Zweck haben, die Schwächeren in die Lage zu versetzen, ihre in der Verfassung verankerten sozialen Grundrechte zu genießen, tritt die philantropische „Wohltätigkeit“, flankiert von einem nahezu anchronistischen Pflichtendiskurs: Wer keine oder auf dem Markt nicht nachgefragte Leistungen erbringt, soll auch keine oder reduzierte und diskriminierende Gegenleistungen erhalten.

Mit dem Übergang von der „welfare“- zur „,workfare“-Philosophie, wie sie exemplarisch von „New Labour“ in Großbritannien vollzogen wird, über die Propagandisten des „Dritten Weges“ aber auch in die Leitvorstellungen kontinentaleuropäischer Sozialdemokraten Eingang gefunden hat, vollzieht sich ein radikaler Bruch in der Grundkonzeption jener Sozialpolitik, die das 20. Jahrhundert in Europa geprägt hat. Soziale Unterstützungsleistungen sollen sich nicht mehr an der Sicherheit (des Einkommens und der Lebensumstände) orientieren sondern an der Nützlichkeit des einzelnen. Ausschlaggebend für den Bezug sozialer Unterstützung im Notfall ist damit nicht länger das „gesellschaftliche Bürgersein“, welches zu legitimen Forderungen nach Sicherheit, Gesundheit und Unterstützung im Alter berechtigt, sondern der Nachweis individueller Produktivität und der (wiederholten) Anstrengungen zur Sicherung von „Beschäftigungsfähigkeit“ („employability“) (vgl. Revelli 1999: 147). Dabei wird die Zugehörigkeit zur Gemeinschaft von einer erbrachten Leistung abhängig gemacht und anders formuliert: auf Rechte soll sich nur derjenige berufen, der seine Pflichten gegenüber der Gemeinschaft erfüllt. Mit dem normativen Selbstverständnis moderner Verfassungsstaaten als einer freiwilligen Assoziation von Rechtsgenossen läßt sich dieser Pflichtendiskurs nur schwer zur Deckung bringen. Denn anders „als in der Moral gelten im Recht die Pflichten als etwas Sekundäres; Pflichten resultieren erst aus der gewünschten Kompatibilität meiner Rechte und den gleichen Rech- 
ten anderer" (Habermas 1998: 817). Und nur wenn, wie im liberalen Denken, den Freiheitsrechten ein Vorrang vor anderen (Wohlfahrts-)Rechten eingeräumt wird, macht es einen Sinn, daß Pflichten von (bedürftigen) Rechtsträgern mit dem Verweis auf die Freiheitsrechte anderer eingeklagt werden, die so die Unterstellung - durch mehr oder weniger großzügige Sozialleistungen an Bedürftige verletzt werden. Dahinter verbirgt sich freilich das Argument, daß leistungsloses Einkommen selbst in einer von struktureller Massenarbeitslosigkeit gebeutetelten Erwerbsarbeitsgesellschaft der normativen Ächtung unterliegen sollte. Wie zuvor im Hinblick auf die Erosion der Leistungsgerechtigkeit ausgeführt, gibt es aber an der Spitze der Einkommenspyramide eine wachsende Zahl von Menschen, die Einkommen (aus Vermögen) beziehen, denen keine Leistungen zugeordnet werden können; sie „lassen das Geld“ - richtig müßte es heißen: andere Menschen - für sich arbeiten und entziehen sich zugleich ihren Pflichten als Steuerstaatsbürger. Kurzum: Der Pflichtendiskurs des „Dritten Weges“ zeichnet sich durch einen eklatanten Mangel an Universalität aus, denn er richtet sich nicht auf alle Rechten- und Pflichtenträger in gleicher Weise.

Wenn sich der Staat aus seiner Funktion Wohlfahrt durch Umverteilung zu stiften, so weit wie irgend möglich zurückzieht und es dem Markt überläßt, für Gerechtigkeit zu sorgen, muß es notwendigerweise zu einem Zuwachs seiner repressiven Aktivitäten kommen. In Gesellschaften mit einer langen sozialstaatlichen Tradition bedarf es eines verstärkten Drucks, damit die Menschen dem Differenzierungsbegehren der Märkte nachgeben. Daher wird es Aufgabe eines „aktivierenden Staates“ die „Teilhabe“ eines jeden am (Arbeits-)Marktgeschehen $\mathrm{zu}$ „stimulieren“ und wenn dies nicht gelingt, eben auch zu erzwingen. Dies kann, wie unter „New Labour“, etwa dadurch geschehen, daß die Aufnahme eines niedrig entlohnten Dienstleistungsjobs durch Sanktionen gegenüber Empfängern von Sozialhilfe oder Arbeitslosenunterstützung erzwungen wird oder dadurch, daß Sozialleistung und individuelle Bedürftigkeit entkoppelt werden und die Unterstützungsleistung an das Einkommen ganzer Familien gebunden wird. Wenn die durch das Kriterium der „Brauchbarkeit“ gesteuerte „Teilhabe“ am Prozeß der ökonomischen Verwertung die einzige Möglichkeit ist, über die Menschen in eine Gesellschaft integriert werden, ist es nur konsequent, das regulative Prinzip der Gleichheit durch das der „Fairness“ zu ersetzen. Die bloße Aussicht auf eine „Teilnahme“ an einem von den Gesetzen der Ökonomie bestimmten Wettbewerb wird zu einer zeitgemäßen Form von Gerechtigkeit.

\section{Flexibilisierung und Beschleunigung bis zum Crash}

Doch drängen sich hier eine Reihe von Fragen auf: Was bedeutet es für die Zukunft der sozialen Demokratie, wenn sich die Schwachen nicht länger auf 
soziale Rechte verlassen können, z. B. darauf, daß sie in gewissem Umfang vor den Leistungszumutungen des Marktes geschützt werden? Welche politischen Konsequenzen sind mit der Erosion der Leistungsgerechtigkeit verbunden? Ist nicht mit „crashs“ zu rechnen, wenn das Tempo des technischökonomischen Wandels die Anpassungsfähigkeit der Menschen überfordert. Oder genauer: Muß nicht mit pathologischen Reaktionen gerechnet werden, wenn die ökonomische Logik, die dazu tendiert, individuelle und gattungsspezifische Eigenzeiten der Menschen ebenso zu vernachlässigen wie die systemspezifischen Zeiten von demokratischen Verfahren, auf alle Handlungsfelder ausgedehnt wird und in alle Lebensbereiche Einzug hält?

Wenn sich nach der CDU/CSU, der FDP und den Bündnisgrünen nun auch die SPD von den Zielsetzungen der Verteilungsgerechtigkeit verabschiedet, welche politische (und mediale) Kraft kann noch die Gerechtigkeitsvorstellungen jener breiten „gesellschaftlichen Mitte“ repräsentieren, deren Angehörige sowohl eine weitestgehende Verteilungsgerechtigkeit als auch eine Staatsverantwortung für entsprechende Umverteilungsmaßnahmen befürworten? $\mathrm{Da}$ dem so ist, dokumentieren nicht zuletzt Befunde aus der milieuorientierten Sozialstrukturforschung und aus der empirischen Gerechtigkeitsforschung.

Die Studien der „Arbeitsgruppe für Internationale Sozialstrukturforschung“ (AGIS) um Michael Vester in Hannover zeigen, daß die „neue gesellschaftliche Mitte" sehr viel mehr Milieus umfaßt als das mit gerade einmal 7\% relativ kleine „postmoderne“ der individualistischen „neuen Aufsteiger“, auf die Medien und die „Modernisierer“ aller Parteien ihr Hauptaugenmerk richten. Die sozialen Milieus der „anderen neuen Mitte“, zu denen Milieus gut ausgebildeter und besonders leistungsorientierter Arbeitnehmer (64\%) und auch große Teile der Bildungselite und der Führungskräfte im öffentlichen Dienst gehören - zusammen stellen sie etwa 83\%-88\% der Befragten - wünschen „den Ausgleich zwischen oben und unten in der Gesellschaft und gleichzeitig Ermunterung der Eigenverantwortung. Sie wollen, arbeitend und Steuer zahlend, etwas leisten, verlangen aber auch Gegenleistungen. Gegen Abstriche sind sie nicht - aber nur wenn sie gerecht begründet sind“" (Vester 1999). Dies unterscheidet sie von den „high potentials“ in avangardistischen Kulturund Medienberufen und in den Unternehmen der „new economy“, die „ihr Bedürfnis nach edlem Konsum, Erlebnis und Erfolg ohne einschränkende Verpflichtung verwirklichen" (ebd.) wollen und daher von einer Programmatik, die auf „marktgenerierte Formen von Gerechtigkeit“ setzt, eher angesprochen werden.

Im Verlauf der wirtschaftlichen Entwicklungen seit Mitte der 70er Jahre und in Reaktion auf die zunehmend restriktive Sozialpolitik unter der KohlRegierung seit den 80er Jahren läßt sich zwar eine leichte Verschiebung von „kollektivistischen Wertorientierungen“ wie „Staatsverantwortung für Sicher- 
heit und Gleichheit“ zugunsten „individualistischer“ Orientierungen wie „Freiheit und Eigenverantwortung" beobachten; von einem sozialkulturellen Wertewandel zugunsten letzterer kann nach Befunden der empirischen Gerechtigkeitsforschung in der Bundesrepublik Deutschland jedoch keine Rede sein (vgl. dazu u. a. Roller 2000). Ganz im Gegenteil: durch die Integration der Bürger der ehemaligen DDR, deren Präferenzen für einen umfassenden Sozialstaat besonders stark ausgeprägt sind, ist die Zahl derer, die eine Stabilisierung des erreichten Niveaus sozialer Sicherung wünschen, eher noch größer geworden. Wird den Bundesbürgern jedoch - im Sinne der liberalen Hypothese eines strukturellen Antagonismus von Freiheit und Gleichheit - in Meinungsumfragen abverlangt, sie sollten sich zwischen persönlicher Freiheit und möglichst großer Gleichheit bzw. sozialer Gerechtigkeit entscheiden, so können die Meinungsforscher des Allensbacher Instituts seit der Bundestagswahl 1998 eine Trendwende nachweisen. Anders als in Ostdeutschland trägt die von der rot-grünen Regierung forcierte Ungleichheitsdebatte in Westdeutschland inzwischen Früchte. Seit für den „Dritten Weg“ gestritten wird, gibt es hier deutlich weniger Stimmen für eine Verringerung sozialer Benachteiligung und für möglichst große Gleichheit (Noelle-Neumann 2000). Zugleich erwartet die übergroße Mehrheit der deutschen Bevölkerung für die nächsten zehn Jahre eine wachsende soziale Differenzierung, mehr Armut und zunehmenden privaten Reichtum, den weiteren Zerfall traditioneller Werte wie Hilfsbereitschaft und Solidarität, mehr soziale Kälte und Egozentrik, vermehrte Spannungen und Unsicherheit.

Anders als in den USA gibt es in Deutschland zwar noch immer eine breite gesellschaftliche Mitte, deren Mitglieder für eine Politik des sozialen Ausgleichs plädieren. Gleichzeitig werden die Angehörigen dieser gesellschaftlichen Mitte durch die Politik der rot-grünen Regierung „nach oben gehoben“ und „nach unten gezogen“ - in das „postmoderne Milieu“ der Börsenspekulanten die einen und in die „postfordistischen Milieus“ der Marginalisierten, der in prekäre und ungeschützte Arbeitsverhältnisse Gedrängten und der zur Zeit noch Gutsituierten, aber ständig vom Absturz Bedrohten die anderen. Die Signale, die sozial abgehängte Arbeitnehmer und Arbeitslose und auch viele derjenigen, die in „prekärem Wohlstand“ leben, aber durch einen alltäglichen Schicksalsschlag (Arbeitslosigkeit, Krankheit, Unfall, Scheidung usw.) unter die Armutsgrenze rutschen können ${ }^{4}$, von den Deutungseliten empfangen, sind ummißverständlich. Vor allem jene 12\% der Bevölkerung, die schlecht qualifiziert sind, sich mit Gelegenheitsjobs und mit Routinearbeiten im Industrie- und Dienstleistungssektor über Wasser halten oder auf Arbeits-

4 Nach einer von Michael Vester zitierten Untersuchung der Caritas leben etwa 20 Millionen Menschen in Deutschland, das entspricht 25\% der Bevölkerung, in „prekärem Wohlstand (Vester 1999). 
losen- oder Sozialhilfe angewiesen sind (Vester 1999), die „Unterprivilegierten“ also, wissen längst, daß sie nichts mehr erwarten dürfen - weder von den Aufsteigern in Wissenschaft, Politik und in den Medien, die ihren Aufstieg der Bildungsexpansion der 60er und 70er Jahre verdanken (oder auch schlicht der Zufälligkeit ihres Erfolgs in der „new economy“), noch von der nach wie vor auf Leistung gepolten „sozialen Mitte“, die selbst von einem Absturz bedroht ist. Wer nicht flexibel, anpassungsfähig und mobil ist, wem es an Schnelligkeit, Bildungsbereitschaft und Findigkeit beim Aufspühren von „Chancen“ mangelt, wer nicht fähig ist, es mit der oft verzweifelten Arbeits- und Leidensfähigkeit von Migranten aufzunehmen und nicht bereit, noch um die schlechtesten Jobs zu konkurrieren als wären es Traumjobs, hat am „Standort Deutschland“ keine Zukunft zu erwarten, muß aus gutem Grunde „Angst vor der Zukunft“ haben. Nach den Erhebungen der neuen Shell-Studie trifft dies auf $80 \%$ aller Hauptschüler zu; sie fühlen sich isoliert, nicht richtig zugehörig zur Gesellschaft (Deutsche Shell 2000).

Wenn alle Parteien auf die vermeintlichen "Sieger" von morgen setzen und sich keiner mehr der „looser“ annehmen will, sind radikale Reaktionen unvermeidlich. Diese werden mit großer Wahrscheinlichkeit rechte sein, in Deutschland wie auf den anderen Wohlstandsinseln, in Österreich, Dänemark, der Schweiz oder in Norditalien. Wenn die Verlierer von Modernisierungs- und Globalisierungsprozessen frühere „Arbeiterparteien“ nicht mehr wählen können (und wohl auch nicht wählen sollen), bleibt ihnen entweder die Stimmenthaltung - wie in den USA, wo ärmere Bevölkerungsgruppen mit einem Jahreseinkommen von unter 10.000 \$ den Präsidentschaftswahlen 1996 mehrheitlich fernblieben (Alber 2000) - oder aber sie votieren für rechtspopulistische Gruppierungen, die Gleichheitsideale, wenn auch in einer deformierten Gestalt, für ihre regressiven Ziele zu instrumentalisieren verstehen. Rechtspopulistische Hexenmeister besetzen die Plätze, die von sozialdemokratischen Alchimisten freigemacht wurden: Wo letztere die Angst der Bürger vor einer linken Alternative in sozialen Fortschritt verwandeln halfen, sorgt heute ein Amalgam aus primitivem Wohlstandschauvinismus, dumpfem Anitsemitismus und aggressiver Fremdenfeindlichkeit dafür, daß aus Verlierern der Globalisierung Verächter der Demokratie werden. Dieses rechtspopulistische Bindemittel füllt die Sinn- und Orientierungslücken, die durch den Verlust sozio-ökonomischer Sicherheit und durch den kollektiven und individuellen Kontrollverlust entstanden sind, der mit Prozessen der ökonomischen wie der kulturellen Entgrenzung einher geht (Heitmeyer 2000). Wenn lebenslange Unsicherheit nahezu das einzig Sichere ist, was die Verlierer von ihrer Zukunft erwarten dürfen, wird verständlich - wenn auch nicht hinnehmbar -, daß sie aggressiv auf dem Wenigen beharren, was ihnen kein noch so beschleunigter Wandel nehmen kann: ihr Deutsch-Sein - und die Gewalt ihrer (männlichen) Fäuste und Waffen. In den „national befreiten Zonen“, die sich 
ostdeutsche Jugendliche von „Fremden“ welcher Art auch immer frei geprügelt haben, lebt es sich zwar keinen Deut besser als zuvor, doch können sich diese Jugendlichen in dem Gefühl wiegen, Kontrolle über ein kleines Stückchen Territorium auszuüben, hier Normen setzen, Maßstäbe für Verhalten vorgeben, Hierarchien etablieren zu können - anstelle der dafür in einer Demokratie vorgesehenen Verfahren und Institutionen.

Doch selbst diejenigen, die sich anstecken lassen vom Zukunftsoptimismus jener Allparteienkoalition der Modernisierer, die leistungsbereiten jungen Menschen, die sich „fit machen“ wollen für die beschleunigte „leichte Moderne“, könnten zu einem Problem werden. Die Mehrheit der deutschen Jugendlichen, so verkünden es die Autoren der Shell-Studie, sei in der Gegenwart angekommen: Sie haben die neoliberalen Flexibilisierungszwänge verinnerlicht. Sie sind bereit, ohne Aussicht auf sozio-ökonomische Sicherheit auf wechselnde Beschäftigungsverhältnisse und alle Anforderungen des Arbeitsmarktes mit Anpassung, Mobilität und ständiger Weiterbildungsbereitschaft zu reagieren. Sie kalkulieren Phasen der Arbeitslosigkeit in ihre Erwerbsbiographie ein und wissen, daß sie die Risiken einer ungesicherten Existenz in wachsendem Umfang individuell zu tragen haben. Eine politische Vision, die über den Tag hinaus reicht, Bilder einer anderen, bessere Gesellschaft haben diese Jugendlichen nicht. Von der Politik erwarten sie gar nichts; daher sind 95\% von ihnen über die CDU-Spendenaffäre auch nicht erschrocken. Nur eine individualisierte Zukunftsvision ist ihnen eigen: die Vorstellung einer Vereinbarkeit von Erwerbsarbeit und Familie. Daß es sich dabei um eine illusionäre Vorstellung handeln könnte, die mit der schönen neuen Flexi-Welt der Arbeitsgesellschaft im 21. Jahrhundert nicht kompatibel ist, kommt ihnen dabei nicht in den Sinn. In dem Maße jedoch, wie sich bei den „flexiblen Fachkräften" von morgen die Einsicht durchsetzt, daß ihre flexible Anpassung an Zwänge des Arbeitsmarktes auf Kosten des privaten Glückes geht und alle Anpassungsbereitschaft daran nichts ändert, daß man die geforderte "Spitzenleistung“ nicht erbringen wird und daher zum abstiegsgefährdeten „Mittelmaß“ gehört, ist mit politischen Reaktionen auch der „sozialen Mitte“ zu rechnen. Dann könnte sich als Bumerang erweisen, daß die morgen nicht mehr so jungen Menschen sich wirklich so sehen, wie es Politiker, Wirtschaftsbosse und Bildungsmanager heute von ihnen erwarten: als Träger von „Humankapital“, das den Schlüssel für Innovation und Wettbewerbsfähigkeit der Wirtschaft darstellt. Als nachgefragte „knowledge worker“ mit „globaler Digitalkompetenz", doch ohne Bildungserfahrungen, die ihnen Widerstandsfähigkeit gegen den mainstream vermittelt hätten. Im besten Falle aber hätten sie noch eine Ahnung von dem, was ein „gutes Leben“ ausmacht.

Spätestens dann wird sich der durch und durch ideologische Gehalt jener These offenbaren, wonach es sich bei der Forderung nach sozialer Gerechtigkeit in Zeiten von Globalisierung und Individualisierung um eine „wirklich- 
keitsfremde“ Vision handelt (Dettling 2000): In einer universalistischen Perspektive, die zugleich geschlechts- und kulturspezifische Besonderheiten zu berücksichtigen versucht, spricht nämlich vieles dafür, Gleichheit nicht mit einem gleichen $\mathrm{Ma}$ an Primärgütern (wie bei Rawls) oder Ressourcen (wie bei Ronald Dworkin) in eins zu setzen. Denn Personen können, abhängig von ihren spezifischen Lebensbedingungen, kulturellen Rahmenbedingungen und ihrer Geschlechtszugehörigkeit, einen ganz unterschiedlichen Gebrauch von diesen Gütern und Ressourcen machen. Amartya Sen (2000) schlägt daher vor, Gleichheit im Sinne von Fähigkeiten („capabilities“) zu verstehen, über die alle Menschen verfügen sollten. Auf diesem Wege läßt sich möglicherweise besser nachvollziehen, in welchem Maß sich Güter und Ressourcen in reale Freiheitsräume transformieren. Für die Frage der Gerechtigkeit wäre demnach ausschlaggebend, welche Fähigkeiten wesentlich sind und welche nicht. Fähigkeiten, wie die, andere verletzen, unterdrücken, manipulieren und täuschen zu können oder Zwang auszuüben, gehören zweifellos nicht zu den Fähigkeiten, die ein Handeln nach Prinzipien ermöglichen, welche für alle gelten. Denn die Anwendung dieser Fähigkeiten setzt gerade voraus, daß andere eben nicht so handeln können. Wohl aber rechnen jene Fähigkeiten, die ein „gutes Leben“ ausmachen, dazu - darunter nicht zuletzt die Fähigkeiten, Bindungen an andere eingehen zu können

Eine Gesellschaft, in der Gegenwärtigkeit alles ist, Vergangenheit und $\mathrm{Zu}$ kunft aber wenig zählen, in der Erwachsene den Kindern keine konsistente Orientierung vermitteln können, weil sie ihr eigenes Leben als Flickenteppich erleben - notdürftig zusammengehalten von Psychotherapeuten, „selfmanagement“, der Tourismus- und der „wellness“-Industrie - wird schwerlich als gerecht wahrgenommen werden können. Denn sie ermöglicht eben keine Gleichheit hinsichtlich der normativen Ansprüche, die an eine „gutes Leben“ gestellt werden können. Dazu gehört, neben der bereits erwähnten Fähigkeit, längerfristige Bindungen eingehen zu können, auch, sein Leben in seinem eigenen Kontext und seiner spezifischen Umgebung führen zu können (vgl. Nussbaum 1998).

Wenn aber gesellschaftliche Bindungen von spontanen ökonomischen Bewegungen nach und nach zersetzt werden, geht es darum, Zeiten und Räume von konkurrenzgetriebener Geschäftigkeit freizuhalten. Ohne diese elementare Voraussetzung können weder Männer noch Frauen jene notwendigen Reproduktionsleistungen erbringen, die nachwachsende Generationen mit dem „emanzipatorischen Minimum“ (O. Negt) ausstatten. Der Wettbewerb auf Märkten und die Eigenlogik von Wissenschaft und Technik, welche sich bekanntermaßen durch normative Blindheit auszeichnen (Latour 1999), werden den Prinzipien der Langfristigkeit und der verminderten Schnelligkeit, ohne die Stabilität auch in modernen Gesellschaften nicht vorstellbar ist, wohl kaum zum Durchbruch verhelfen. Hier ist und bleibt die Politik gefordert. 


\section{6. Über die befreiende Wirkung des Rechts}

Ein Weg in die Zukunft, der soziale Gerechtigkeit als wahrscheinliche Folge ökonomischen Wachstums konzipiert, ist weder realistisch noch wünschenswert. Einmal ganz abgesehen davon, daß die ökologischen Voraussetzungen exponentiell gesteigerten Wachstums und damit zugleich die Probleme der „globalen Gerechtigkeit“ vollständig ausblendet werden: Es gibt auch gute Gründe, die ökonomische Tragfähigkeit dieser Vision anzuzweifeln.

Die Idee eines kurz bevorstehenden Booms, der uns schon in naher Zukunft ein beschäftigungswirksames Wachstum bescheren soll, ist inspiriert durch die von den Börsen und den Medien hochgejubelte „new economy“. Insbesondere der Boom, den neu gegründete Internet-Unternehmen in den USA erleben, hat die Fiktion eines neuen ökonomischen Paradigmas entstehen lassen. Es ist dies die Fiktion eines weitgehend krisenfreien Wachstums, ganz ohne Inflation, ausgelöst durch exorbitante Produktivitätssteigerungen auf der Basis der neuen Technologien, die Lohnerhöhungen und gleichzeitig Stückkostenreduktionen möglich und daher Verteilungskonflikte überflüssig machen. Daß es sich dabei in der Tat mehr um Wunschdenken denn um einen Königsweg aus dem seit mehr als 20 Jahren bekannten Dilemma von „Wachstum ohne Wohlstand“ oder „Wachstum ohne Beschäftigung“ handelt (vgl. dazu Altvater/Mahnkopf 2000), könnte ein etwas genauerer Blick auf das „Modell USA“ verdeutlichen. Denn am US-amerikanischen Fall läßt sich exemplarisch studieren, daß auch die funktionelle Ungleichheit mit sozialen und ökonomischen Kosten verbunden ist. Dies sind vor allem diejenigen Kosten, die für öffentliche und private Sicherheit anfallen. Denn die Herrschaft eines Gesellschaftsmodells, das auf soziale Ungleichheit als seinen „sportlichen Motor“ setzt, muß durch ständige Repression abgesichert werden. Ein zum „workfare“ degenerierter Wohlfahrtsstaat bedarf der Absicherung durch „law and order“ - zumal in jenen innerstädtischen Zonen, in denen die „überflüssige Restbevölkerung“ lebt ${ }^{5}$-, der elektronischen Überwachung öffentlicher Räume und einer Instrumentalisierung des Strafrechts für arbeitsmarktpolitische Zwecke (Western/ Beckett 1998). Insgesamt betrachtet dürften die Kosten größerer Ungleichheit wohl nicht geringer sein als die hierzulande zum „Standortnachteil“ stilisierten Kosten eines vor- und nachsorgenden Sozialstaats. Außerdem könnte ein etwas genauerer Blick in die USA helfen, hinter dem dortigen „Beschäftigungswunder“ Defizite der US-

5 Ein überaus lesenswerter Beitrag über „Amerikas Ghettos“ in einer Julinummer der „Frankfurter Allgemeinen Zeitung“ beginnt mit den Sätzen: „Wären die amerikanischen Großstadtghettos eine eigene Nation, dann ginge es hier nicht bloß um soziale Probleme. Dann sprächen wir von einem Bürgerkrieg. Dann wäre der Tod von Amadou Diallo in der Bronx kein 'tragischer Tod' gewesen, sondern eine standrechtliche Hinrichtung; und die Prügel für Rodney King, deren Videoaufzeichnungen 1992 die Los Angeles Riots auslösten, wäre kein Übergriff der Polizei, sondern staatlich sanktionierte Folter" (Kreye 2000). 
amerikanischen Ökonomie und Gesellschaft zu erkennen, die wir zum Glück in Europa (noch nicht) beklagen müssen (vgl. Schäfer 2000b). Es gibt ökonomische Grenzen für die absoluten Zuwächse des Wachstums, die mit dem bereits erreichten Wachstumsniveau in den Industrieländern zu tun haben; daran wird auch die „new economy“ nichts ändern. Was sich indes verändern wird, das ist das bislang noch niedrige Produktivitätsniveau von Dienstleistungstätigkeiten. Wenn in Zukunft, wie in der „alten Ökonomie“ in Vergangenheit und Gegenwart, die Produktivitätszuwächse die Zuwachsraten des Wachstums übersteigen, so ist mit einer weiteren Reduktion des Arbeitsvolumens zu rechnen.

Sehr viel grundsätzlicher noch muß aber auch danach gefragt werden, wie sich ein Konzept, daß die Herstellung sozialer Gerechtigkeit zu einer erwünschten, aber keineswegs garantierbaren Nebenfolge ökonomischen Wachstums macht, mit Zielsetzungen globaler Gerechtigkeit und ökologischer Nachhaltigkeit verträgt. Können wir für uns, hier in Europa und in den anderen Industrieländern, wirklich ein dauerhaftes Wachstum von 3 oder 4 Prozent wünschen? Das entspräche, auf die nächsten 60 Jahre gesehen, einer Verzehnfachung der Produktion, mit einem entsprechend höheren Verbrauch an physischen Ressourcen und der Emission von Schadstoffen, es sei denn, beschäftigungsschaffendes zukünftiges Wachstum wäre vorstellbar ohne materielle Produkte, Transport und Verbauch an natürlichen Ressourcen. Weil dies trotz aller Ansätze zur Steigerung von Energieeffizienz bloßes Wunschdenken ist und weil die natürlichen Ressourcen endlich sind, begrenzt weiteres Wachstum bei uns die Chancen des Wachstums in den Ländern des Süden. Dies ist aber nicht nur ungerecht, weil es die ökonomische und gesellschaftlichen Entwicklung gerade in diesen Ländern blockiert, es ist noch nicht einmal ökonomisch. Denn mit der Zunahme globaler Ungleichverteilung von Ressourcen und Lebenschancen verschärfen sich zugleich soziale und politische Konflikte, Bürger- und Ressourcenkriege sind daher unausweichlich. Daß dabei ganz profane finanzielle Kosten auch für uns im sicheren Deutschland fallen, hat zuletzt der Kosovo-Krieg der NATO bewiesen: Mit den 10 Milliarden, die allein die Bundesrepublik Deutschland dafür aufbringen mußte, wären wohl eine ganze Reihe von „friedensstiftenden Maßnahmen" finanzierbar gewesen. Sicherheitspolitik anstelle von Sozialpolitik ist daher keineswegs billiger. So läßt sich die These aufstellen: Betrachtet aus der Perspektive ganzer Gesellschaften - und nicht etwa aus der von einigen wenigen Marktakteuren - rechnet sich (noch mehr) Ungleichheit nicht, von ethischen Prinzipien und von einem gemeinsamen Interesse an der Sicherung des Friedens in einer kleiner gewordenen Welt einmal ganz zu schweigen.

Mit Eric Hobsbawm aber läßt sich auch eine zweite These formulieren: Weil sich unsere zentralen Probleme zukünftig über Wachstum nicht mehr lösen lassen, wird die Verteilung zur Kernfrage des 21. Jahrhunderts und für lange 
Zeit wird sie wohl um das Problem von öffentlicher Armut und privatem Reichtum kreisen. Was wir unter einer zeitgemäßen Form von sozialer Gerechtigkeit verstehen wollen, kann nur in Prozessen demokratischer Willensbildung (und nicht in den Kreisen liberaler Vordenker), in der politischen Öffentlichkeit und in den Parlamenten ausgehandelt werden. Doch es ist mehr als unwahrscheinlich, daß Verteilungsgerechtigkeit in solchen Prozessen eine zweit- oder gar drittrangige Rolle spielen wird. Freilich läßt sich Verteilungsgerechtigkeit zukünftig nicht mehr an der steigenden Verfügbarkeit von Konsumgütern für alle bemessen. Es wird auch nötig sein, neue, den Bedingungen einer entgrenzten Ökonomie angemessene Bezugsgrößen für die Ermittlung von Leistungsgerechtigkeit zu verhandeln. Das Normalarbeitsverhältnis und die darauf auflagernden Institutionen, einschließlich des auf den fordistischen „employment contract“ bezogenen „gender contracts“, wird sicherlich keine normative Basis für die Ermittlung eines allgemein akzeptierten Maßstabs für Leistungen und Gegenleistungen abgeben. Dies vor allem auch deswegen nicht, weil in der liberalen Version von Leistungsgerechtigkeit immer nur Zwecktätigkeiten auf (knapper werdenden) Funktionsstellen mit einem „gerechten Lohn“ bedacht wurden. Für Leistungen, die (in der Regel unentgeltlich) von Frauen außerhalb des Arbeitsmarktes erbracht werden, hatte dieses Prinzip von Leistungsgerechtigkeit nie größere Bedeutung.

Geändert hat sich vermutlich nicht so sehr das gesellschaftliche Bedürfnis nach Gerechtigkeit - wohl aber die Begründung, die zu Beginn des 21. Jahrhunderts für diese regulative Ideal von Gesellschaftspolitik möglich ist. An die christliche „Brüderlichkeit aller Menschen“ mag heute kaum jemand appellieren, schloß diese doch schon in der Vergangenheit weit mehr Menschen aus als sie einbezogen hat. Doch auch die Schicksal- und Solidargemeinschaft der Nation, die unter dem Druck der globalen Konkurrenz ihre Bindekraft verliert, scheint für Fragen sozialer Gerechtigkeit immer weniger ansprechbar $\mathrm{zu}$ sein und selbst die internationale Arbeiterbewegung getraut sich heute kaum mehr an soziale Gerechtigkeit zu appellieren. In den reichen Industrieländern hat der „american way of life“ die verpflichtenden sozialmoralischen Milieus zersetzt, mit der Folge, daß (arbeiter)kulturspezifisches „Mitleiden“ heute immer weniger Menschen dazu bewegt, sich ,in die Reihen einzureihen“, nur weil sie „auch Arbeiter sind“. In den Transformationsländern, in den Entwicklungs- und Schwellenländern gibt es eine wachsende Zahl von Menschen, die im Schattenreich der „Informalität“ ihre Existenz zu sichern versuchen; Lohnarbeiter sind sie nur in Ausnahmefällen oder für kurze Phasen in ihrem Leben - und daher von den Organisationen der Arbeiterbewegung, selbst wenn diese dies ernsthaft versuchten, kaum noch erreichbar. Damit gibt es nur noch einen universalen Begründungszusammenhang für soziale Gerechtigkeit, nämlich jenen, der durch den Menschenrechtsdiskurs eröffnet wurde. Freilich muß dieser zuerst aus den Höhen der Ideale in die 
Niederungen institutioneller Verpflichtungen gelangen. Voraussetzung dafür wäre womöglich, daß in den westlichen Industrieländern - wo die Pflichtenträger, die den Menschenrechten zuzuordnen sind, in Sonderheit gesucht werden müssen -, ein Rückbezug auf den (bürgerlichen) Begriff des „guten Lebens" stattfindet. Der Bildung kommt dabei tatsächlich eine Schlüsselrolle $\mathrm{zu}$; doch handelt es sich hier um eine andere als die von den „neuen Sozialdemokraten" beschworene. Es geht um die Entfaltung all jener menschlicher Fähigkeiten und Kompetenzen, die zu einem „guten Leben“ gehören; sie wären eine wichtige Voraussetzung: um eine breite gesellschaftliche Debatte über die wünschenswerte Entwicklung von Wirtschaft und Wissenschaft in Gang zu setzen; für gesellschaftliche Arrangements, die aus der Schwäche anderer keinen Vorteil zu ziehen trachten; für die Entstehung und Befestigung von (globalen) Institutionen, die die Machtlosigkeit und Verletzbarkeit (von einzelnen Menschen und ganzen Gesellschaften) reduzieren.

Um Verteilungsgerechtigkeit unter den Bedingungen offener Grenzen und eines begrenzten Umweltraumes zu reformulieren, muß daran erinnert werden, daß Gleichheit und Freiheit nur aus der verzerrten Weltsicht eines Neoliberalen Gegensätze sind. Wenn unter Freiheit mehr als nur die Abwesenheit von Zwang verstanden wird, nämlich die Möglichkeit, ein Leben nach eigenen Entscheidungen, eine frei gewählte Existenz zu führen, erweist sich sogleich die Kontrolle über die eigenen Existenzbedingungen, darin eingeschlossen die sozialen Einrichtungen zur Erweiterung individueller Freiheiten und die politische Partizipation als konstitutive Bedingungen von Entwicklung (vgl. dazu auch Ladwig in diesem Heft) als der neuralgische Punkt, an dem Freiheit ohne eine Verringerung von Ungleichheit, Machtlosigkeit und Verletzbarkeit zur Schimäre wird (vgl. dazu Sen 2000). Unter Ungleichen kann Gerechtigkeit nichts anderes bedeuten als eine Verpflichtung, die Möglichkeiten der mächtigen Akteure zu verringern und zu zügeln und die von verletzbaren Akteuren zu erweitern (O'Neill 1998: 229f). Daher kann es nicht gerecht sein, wenn die Politik eine wirtschaftliche Dynamik befördert, welche Menschen im ökonomischen Sinne überflüssig macht und wenn die Effizienzsteigerung über alle anderen politischen Ziele gestellt wird.

Weil zwischen Starken und Schwachen „es die Freiheit ist, die unterdrückt und das Gesetz, das befreit“ (J.J. Rousseau), wird es wohl auch morgen darum gehen, daß die Politik in globalen Räumen wie in nationalen der Marktdynamik Grenzen setzt - und im Inneren der Gesellschaften „mehr Demokratie wagt“. Aufgabe der Politik bleibt es auch unter den Bedingungen der Globalisierung, die Macht insbesondere solcher Akteure zu kontrollieren und zu steuern, die sich eine eigene rechtliche Welt geschaffen haben. Müßte nicht auch, so spekuliert ein Verfassungstheoretiker in der „Neuen Züricher Zeitung", das Ideal des 'Bürgerstatus' auf den Funktionskern eines Unternehmens projiziert werden? 
„Es liesse sich etwa die Vorstellung entwickeln, dass Unternehmen in Zukunft einmal (....) ähnlich wie Städte und Dörfer und nicht mehr als blosse Apparate gesehen würden und sie vielleicht auf einer eigenen 'Verfassung' beruhten: einer ' Verfassung', welche die Mitarbeiter, aber auch etwa Konsumenten, Investoren und Lieferanten als eine Art 'Bürger' mit ihren Rechten und Pflichten verstände, Transparenz und eine Art öffentliche Rechenschaft statuierte, gewaltenteilige, vielleicht föderative Strukturen vorsähe, ja sogar Standards zur Wahrung elementarer sozialer und ökologischer Interessen der zeitgenössischen Generation wie auch künftiger Generationen enthielte“ (Thürer 2000).

Auch wenn sich der Tiger nicht zum Vegetarier macht läßt, muß doch der Versuch unternommen werden, „unverfaßte“ ökonomische Mächte, die nicht der effektiven Kontrolle eines einzelnen Staates unterstellt sind, in umfassende Ziele und Prinzipien von Recht und Gerechtigkeit einzubetten. Wenn sich das Letztentscheidungsrecht der Bürger über die wirtschaftliche und gesellschaftliche Entwicklung nicht verteidigen läßt, steht der Demokratie eine düstere Zukunft bevor. Nicht anders als in den Anfängen der Arbeiterbewegung sind die soziale und die demokratische Frage auch unter den Bedingungen eines globalen Kapitalismus aufs engste miteinander verknüpft.

\section{Literatur}

Alber, Jens (2000): Warum die meisten Amerikaner nicht wählen, einige aber doch: Zur historischen Entwicklung der Wahlbeteiligung bei amerikanischen Präsidentschaftswahlen, in: Leviathan, J. 28, H. 3 (2000): 319-342.

Altvater, Elmar (2000): Wirtschaftliches Wachstum, globale Finanzkrise und ökologische Nachhaltigkeit - Ein wirtschaftspolitisches Trilemma, in: Gewinne ohne Menschen. Frühjahrsplenum der Europäischen Akademie der Wissenschaften und Künste, Berlin, 28.-29. Mai 1999, Bern: Schweizerische Akademie der Geistes- und Sozialwissenschaften: 57-87.

Altvater, Elmar/ Mahnkopf, Birgit (1999): Grenzen der Globalisierung. Ökonomie, Ökologie und Politik in der Weltgesellschaft, Münster, 4. völlig überarbeitete Auflage (Verlag Westfälisches Dampfboot).

Altvater, Elmar/ Mahnkopf, Birgit (2000): „New Economy“ - nichts Neues unter dem Mond?, in: WSI-Mitteilungen, Jg. H. 12 (2000)(im Erscheinen).

Aristoteles (1991): Die Nikomachische Ethik, Müchen (dtv).

Bauman, Zygmunt (1999): Die Moderne als Geschichte der Zeit, in: Jahrbuch Arbeit und Technik 1999/2000: „Was die Gesellschaft bewegt“, hrsg. von W. Fricke, Bonn (Verlag J.H.W. Dietz Nachf.): $19-30$.

Blomert, Reinhard (1999): Hohepriester der Unternehmenskultur, in: Berliner Zeitung vom 23./24.Oktober 1999.

Boltanski, Luc/ Chiapello, Ève (2000): Befreiung vom Kapitalismus? Befreiung durch Kapitalismus? in: Blätter für deutsche und internationale Politik, H. 4 (2000): 476-497.

Bourdieu; Pierre/ Wacquant, Luc (2000): Schöne neue Begriffswelt, in: Le monde diplomatique, Mai 2000: 7.

Clement, W. (2000), Durch innovative Politik zu gerechter Teilhabe. Rede anläßlich des Forums Grundwerte: Gerechtigkeit“ der SPD in Berlin am 26.April 2000; http://www.spd.de/events/grundwerte/clement.html.

Daly, Herman E. (1999): Wirtschaft jenseits von Wachstum. Die Volkswirtschaftslehre nachhaltiger Entwicklung, Salzburg/ München (Verlag Anton Pustet).

Dettling, Winfried (2000): Gleichheit - Vielfalt - Fairness, in: www.boell.de 1 (2000): 10-11.

Deutsche Shell (Hg.)(2000): Jugend 2000. Gesamtkonzeption und Koordination: A. Fischer/ Y. Fritsche/ W. Fuchs-Heinritz/ R. Münchmeier, 2 Teilbd., Opladen (Leske + Buderich Verlag).

DIW (Deutsches Institut für Wirtschaftsforschung)(2000): Einkommensverteilung in Deutschland - Stärkere Umverteilungseffekte in Ostdeutschland, Wochenbericht 19/2000: 291-297.

Ehrke, Michael (1999): Der Dritte Weg und die europäische Sozialdemokratie. Ein politisches 
Programm für die Informationsgesellschaft?, Bonn: Friedrich-Ebert-Stiftung.

Fracer, Nancy (1998). Social Justice in the Age of Identity Politics: Redistribution, Recognition, Participation, discussion paper FS I 98-108, Wissenschaftszentrum Berlin für Sozialforschung.

Giddens, Anthony (1999a): „Der Dritte Weg macht Hoffnung“, Interview in: DIE WELT vom 18. Oktober 1999.

Giddens, Anthony (1999b): Der dritte Weg. Die Erneuerung der sozialen Demokratie, Frankfurt/M. (Suhrkamp).

Giddens, Anthony (2000): Die Politik des Dritten Weges, in: H. Flassbeck/ A. Giddens/ F. Wiethold/ K. Zwickel u. a., Ein dritter Weg in das dritte Jahrtausend. Von der Standort- zur Zukunftsdebatte, Hamburg (VSA-Verlag): 12-25.

Glotz, Peter (1999): Die beschleunigte Gesellschaft. Kulturkämpfe im digitalen Kapitalismus, München (Kindler Verlag).

Gorz, André (2000): Eine ganz andere Weltzivilisation denken, in: Blätter für deutsche und internationale Politik, H. 5 (2000): 607-617.

Habermas, Jürgen (1998): Die postnationale Konstellation und die Zukunft der Demokratie, in: Blätter für deutsche und internationale Politik, Nr. 7 (1998): 805-817.

Hank, Rainer (2000): Das Ende der Gleichheit, in: Der Tagesspiegel vom 30.April/ 1. Mai 2000.

Heitmeyer, Wilhelm (2000): Kontrollverluste und Bedrohungsgefühle. Anonymität und Alternativlosigkeit führen zu autoritären Versuchungen, in: Frankfurter Rundschau, Dokumentation vom 6. Mai 2000 (Teil 1) und vom 8. Mai 2000 (Teil 2).

Hobbes, Thomas (1839/1959): Vom Menschen (de homine) - Vom Bürger (de cive), Hamburg.

Hoffritz, Jutta (2000): Ungleichheit in deutschen Chefetagen, in: DIE ZEIT vom 29. Juni 2000.

Kersting, Wolfgang (1998): Der Markt - das Ende der Geschichte? in: B. P. Priddat u.a., Homo oeconomicus: Der Mensch der Zukunft?, Stuttgart/Berlin/Köln (Verlag W. Kohlhammer): 93129.

Kreye, Andrian (2000): Die aufrechte Gang. Philadelphia und anderswo: Amerikas Ghettos geben keine Ruhe, in: Frankfurter Allgemeine Zeitung vom 18. Juli 2000.

Kunz, Harry (2000): Die Zukunft des Sozialen, in: Kommune, H. 8 (2000): 45-50.

Lang, Klaus (2000): Arbeit - Innovation - Gerechtigkeit, in: metall debatte, Nr. 1 (2000): 4-25.

Lefort, Claude (2000): Die demokratische Gesellschaft ist keine Gesellschaft von Individuen, in: Kommune, H. 3 (2000): 49-53.

Mahnkopf, Birgit (1999 b): Von den Risiken beschleunigten Wandels - oder: Grenzen der Flexibilisierung im Unternehmen, in: PASS IT-Consulting Group (Hg.), Wandel als Herausforderung und Chance. Beiträge zum Management von Innovationen, Aschaffenburg: 110- 143.

Mahnkopf, Birgit (1999a): Between the Devil and the Deep Blue Sea: The „German Model“ under the Pressure of Globalisation, in: L. Panitch/ Leys, C. (eds.), Global Capitalism versus Democracy. Socialist Register 1999, London (Merlin)/ New York (Monthley Review)/ Halifax (Fernwood): 142-177.

Mahnkopf, Birgit (2000): Viele Wege führen ins dritte Jahrtausend, in: H. Flassbeck/ A. Giddens/ F. Wiethold/ K. Zwickel u. a., Ein dritter Weg in das dritte Jahrtausend. Von der Standort-zur Zukunftsdebatte, Hamburg (VSA-Verlag): 26-41.

Marx, Karl (1875): Kritik des Gothaer Programms, MEW Bd. 19.

Merkel, Wolfgang (2000): Soziale Gerechtigkeit in Zeiten der Globalisierung. Begründung für eine sozialdemokratische Chancenverteilungspolitik, Vortrag auf einer Tagung der FriedrichEbert-Stiftung und des Netzwerks Berlin unter dem Titel „Flexibilisierung und Sicherheit“ am 4. Juni 2000 in Freudenstadt.

Müller-Plantenberg, Urs (1998): Zukunftsverbrauch. Probleme intergenerationaler Verteilung und sozialer Gerechtigkeit, in: M. Heinrich/ D. Messner (Hg.), Globalisierung und Perspektiven linker Politik, Münster (Westfälisches Dampfboot): 321-340.

Neckel, Sighard (2000): Leistung versus Erfolg. Der Zufall von Reichtum und Ruhm, in: Frankfurter Rundschau vom 7. Oktober 2000.

Noelle-Neumann, Elisabeth (2000): Färben ideologische Positionen in Deutschland von Ost nach West ab? In: Das Parlament Nr. 37/38 vom 8./15. September 2000.

Nussbaum, Martha C. (1998): Gerechtigkeit oder Das gute Leben, Frankfurt/M. (Suhrkamp Verlag).

O' Neill (1998): Transnationale Gerechtigkeit, in: St. Gosepath/ G. Lohmann (Hg.), Philosophie der Menschenrechte, Frankfurt/Main (Suhrkamp Verlag): 188-232. 
Rawls, John (1991): Eine Theorie der Gerechtigkeit, Frankfurt/M. 6. Auflage (Suhrkamp) Verlag.

Revelli, Marco (1999): Die gesellschaftliche Linke. Jenseits der Zivilisation der Arbeit, Münster (Verlag Westfälisches Dampfboot).

Ritsert, Jürgen (997): Gerechtigkeit und Gleichheit, Münster (Verlag Westfälisches Dampfboot).

Roller, Edeltraud (1999): Staatsbezug und Individualismus: Dimensionen des soziokulturellen Wertewandels, in: Th. Ellwein/ E. Holtmann (Hg.), 50 Jahre Bundesrepublik Deutschland. Sonderheft 30 der Politischen Vierteljahresschrift, Opladen (Westdeutscher Verlag): 229-246.

Schäfer, Claus (Hg.)(2000a): Geringe Löhne - mehr Beschäftigung?, Hamburg (VSA-Verlag).

Schäfer, Claus (2000b): Soziale Gerechtigkeit, Wachstum und Nachhaltigkeit nach dem sozialdemokratischen Zeitalter, in: E. Altvater/ B. Mahnkopf (Koord.), Ökonomie eines friedlichen Europa. Ziele - Hindernisse - Wege, Schriftenreihe des Österreichischen Studienzentrums für Frieden und Konfliktlösung - ÖSKF (HG.), Studien für europäische Friedenspolitik Bd. 6, Münster (agenda Verlag): 119-138.

Scharping, Rudolf (2000): Freiheit als sozialdemokratischer Grundwert. Rede anläßlich des Forums Grundwerte: Gerechtigkeit“" der SPD in Berlin am 26.April 2000; http://www.spd.de/ events/grundwerte/freiheit/scharping.html.

Seidel, Eberhard (2000): Das Versagen der Aufsteiger, in: Die Tageszeitung vom 19./20. August 2000.

Sen, Amartya (2000): Ökonomie für den Menschen. Wege zu Gerechtigkeit und Solidarität in der Marktwirtschaft, München/ Wien (Hanser Verlag).

Siebert, H. (2000), Neue Impulse für die neue Ökonomie; in: Frankfurter Allgemeine Zeitung vom 20. Mai 2000.

Strasser, Johanno (2000): Triumph der Selbstdressur, in: Süddeutsche Zeitung vom 16. September 2000 .

Streeck, Wolfgang (2000): Die Bürgergesellschaft als Lernzielkatalog, in: Die Mitbestimmung, Jg. 46, H. 6 (2000): 28-31.

Thürer, Daniel (2000): Globalisierung - Wirtschaftsmacht und Menschenrechte, in: Neue Züricher Zeitung vom 10. Juni 2000.

Urban, Peter (2000): Kneifen gilt nicht, in: metall debatte, Nr.1 (2000): 27-31.

Vester, M. (1999), Gibt es eine 'neue Mitte'? Die gesellschaftliche Basis für eine sozialdemokratische Reformpolitik, Beitrag auf der Konferenz „Modernisierung der Sozialdemokratie“ am 28.11.1999 in Berlin.

Western, B./ Beckett, K. (1998), Der Mythos des freien Marktes. Das Strafrecht als Institution des US-amerikanischen Arbeitsmarktes, in: Berliner Journal für Soziologie 2/1998, S. 159-180.

Wiethold, Franziska (2000): Giddens' Welt der 'harmonischen Widersprüche' in: H. Flassbeck/ A. Giddens/ F. Wiethold/ K. Zwickel u. a., Ein dritter Weg in das dritte Jahrtausend. Von der Standort- zur Zukunftsdebatte, Hamburg (VSA-Verlag): 42-52.

Zeuner, Bodo (2000): Das System Schröder/Fischer oder die unvollständige Abschaffung der Politik, in: Blätter für deutsche und internationale Politik, H. 10 (2000): 1185-1196.

Zilian, Hansgeorg (2000): Taylorismus der Seele, in: Österreichische Zeitschrift für Soziologie, H. 2 (2000): 75-97. 